\title{
THE LIMIT BEHAVIOR OF ELEMENTARY SYMMETRIC POLYNOMIALS OF I.I.D. RANDOM VARIABLES WHEN THEIR ORDER TENDS TO INFINITY ${ }^{1}$
}

\author{
BY PÉTER MAJOR
}

\author{
Hungarian Academy of Sciences
}

\begin{abstract}
Let $\xi_{1}, \xi_{2}, \ldots$ be a sequence of i.i.d. random variables, and consider the elementary symmetric polynomial $S^{(k)}(n)$ of order $k=k(n)$ of the first $n$ elements $\xi_{1}, \ldots, \xi_{n}$ of this sequence. We are interested in the limit behavior of $S^{(k)}(n)$ with an appropriate transformation if $k(n) / n \rightarrow \alpha, 0<\alpha<1$. Since $k(n) \rightarrow \infty$ as $n \rightarrow \infty$, the classical methods cannot be applied in this case and new kinds of results appear. We solve the problem under some conditions which are satisfied in the generic case. The proof is based on the saddlepoint method and a limit theorem for sums of independent random vectors which may have some special interest in itself.
\end{abstract}

1. Introduction. In this paper the following problem is investigated: let $\xi_{1}, \ldots, \xi_{n}$ be i.i.d. random variables with some nondegenerate distribution function $F(x)$; that is, we assume that the distribution of the random variables $\xi_{j}, j=1, \ldots, n$ is not concentrated in a single point. Define the elementary symmetric polynomials,

$$
S^{(k)}(n)=S^{(k)}\left(n, \xi_{1}, \ldots, \xi_{n}\right)=\sum_{1 \leq i_{1}<i_{2}<\cdots<i_{k} \leq n} \xi_{i_{1}} \cdots \xi_{i_{k}} .
$$

We are interested in the limit behavior of the random variables $S^{(k)}(n)$ if $n \rightarrow \infty, k=k(n)$ and $\alpha(n) \rightarrow \alpha^{*}, P(\xi=0)<\alpha^{*}<1$, where $\alpha(n)=1-k(n) / n$. The expression defined in (1.1) is a special $U$-statistic of order $k$.

The limit behavior of $U$-statistics for fixed $k$ is fairly well understood (see, e.g., [1]). These results imply in particular that if $E \xi=0$, then for fixed $k$ the random variables $n^{-k / 2} S^{(k)}(n)$ have a limit distribution which can be expressed by means of a $k$-fold multiple Wiener integral. But in our case the number $k=k(n)$ tends to infinity simultaneously with $n$. Hence the classical results cannot be applied, and a different kind of limit theorems appears. The problem we discuss here was investigated in earlier papers in some special cases (see [2], [3] and [6]). In paper [2] a law of large numbers was proved if the random variables $\xi_{j}$ are nonnegative, and in paper [3] the limit behavior of $S^{(k)}(n)$ was described in the special case when $P\left(\xi_{j}=1\right)=P\left(\xi_{j}=-1\right)=1 / 2$. Paper [6] contains a generalization of paper [3] when the distribution of $\xi_{j}$ is concentrated in three point, 0 and \pm 1 , and $P\left(\xi_{j}=1\right)=P\left(\xi_{j}=-1\right)=$ $1 / 2 P\left(\xi_{j} \neq 0\right)$. But the method of this paper is not strong enough to handle more general distributions.

Received September 1997; revised June 1998.

${ }^{1}$ Supported in part by the Hungarian OTKA Foundation T26176.

AMS 1991 subject classifications. Primary 60F05; secondary 60B15.

Key words and phrases. Limit theorems, $U$-statistics, saddlepoint method. 
The proof of the above papers was based on the saddlepoint method. In this paper also this method is applied. Several technical difficulties had to be overcome to make this method work in the general case. It shows a strong similarity with the technique applied in the theory of large deviations.

We also want to understand whether the limit distribution of the appropriately transformed statistics $S^{(k)}(n)$ shows some universality, that is, whether it depends only on $\alpha^{*}=\lim _{n \rightarrow \infty} \alpha(n)$ or it strongly depends on the sequence $k(n)$ and the distribution function $F(x)$ of the random variables $\xi_{j}$. We prove that in the generic case, although the normalization depends on $\alpha(n)$, the limit distribution depends only on $\alpha^{*}$.

The investigation is based on the following observation. Define the polynomial

$$
Z_{n}(x)=Z_{n}\left(x, \xi_{1}, \ldots, \xi_{n}\right)=\prod_{j=1}^{n}\left(x+\xi_{j}\right)
$$

Then

$$
Z_{n}(x)=\sum_{k=1}^{n} S^{(k)}(n) x^{n-k}
$$

hence

$$
\begin{aligned}
S^{(k)}(n)= & \frac{1}{(n-k) !} \frac{d^{(n-k)}}{d x^{(n-k)}} Z_{n}(x)=\frac{1}{2 \pi i} \oint_{|\zeta|=r} \frac{Z_{n}(\zeta)}{\zeta^{n-k+1}} d \zeta \\
= & \frac{1}{2 \pi} \int_{-\pi}^{\pi} \frac{\prod_{j=1}^{n}\left|r e^{i \varphi}+\xi_{j}\right|}{r^{n-k}} \\
& \quad \times \exp \left\{-i(n-k) \varphi+i \sum_{j=1}^{n} \arg \left(r e^{i \varphi}+\xi_{j}\right)\right\} d \varphi
\end{aligned}
$$

for arbitrary $r>0$. We investigate the expression $S^{(k)}(n)$ in the form defined in (1.2). To handle this integral it is natural to choose the constant $r$, the radius of the circle where the integration is taken, in the way the saddlepoint method suggests. Hence it is natural to look for a point $(r, \bar{\varphi})=$ $\left(r\left(\xi_{1}, \ldots, \xi_{n}\right), \bar{\varphi}\left(\xi_{1}, \ldots, \xi_{n}\right)\right)$ where the partial derivatives of the (random) expression

$$
\sum_{j=1}^{n} \log \left|r e^{i \varphi}+\xi_{j}\right|-(n-k) \log r
$$

disappear. In papers [2], [3] and [6] such an approach was applied. We shall slightly modify this method by looking for an approximative solution, for an asymptotic but nonrandom approximation of the saddlepoint. The laws of large numbers suggests that

$$
\sum_{j=1}^{n} \log \left|r e^{i \varphi}+\xi_{j}\right| \sim n E \log \left|r e^{i \varphi}+\xi\right|=n H(r, \varphi)
$$


with

$$
H(r, \varphi)=H(z)=E \log \left|r e^{i \varphi}+\xi\right|=\frac{1}{2} E \log \left(r^{2}+\xi^{2}+2 r \xi \cos \varphi\right),
$$

where $\xi$ is an $F$-distributed random variable and $z=r e^{i \varphi}$. Because of the parity properties of the integral at the right-hand side of (1.3) it is enough to look for the (asymptotic) saddle point for $0 \leq \varphi \leq \pi$, that is, for a solution in the upper half-plane. We will show that under general conditions there is a point $(r, \bar{\varphi}), \bar{\varphi}=\bar{\varphi}(r)$, such that the relations

$$
\frac{\partial}{\partial \varphi}[H(r, \bar{\varphi})-\alpha(n) \log r]=0, \quad \frac{\partial}{\partial r}[H(r, \bar{\varphi})-\alpha(n) \log r]=0
$$

hold. We rewrite these equations in the equivalent form

$$
\frac{\partial}{\partial \varphi} H(r, \varphi)=\left.0\right|_{\varphi=\bar{\varphi}},\left.\quad r \frac{\partial}{\partial r} H(r, \varphi)\right|_{\varphi=\bar{\varphi}}=\alpha(n),
$$

and also require that the solution $(r, \bar{\varphi})$ satisfy the relation

$$
\bar{\varphi} \text { is the place of maximum of } H(r, \varphi) \text { (as a function of } \varphi, 0 \leq \varphi \leq \pi \text { ). }
$$

Let us remark that the solution of (1.4) [together with the property (1.5)] depends on $n$ through the function $\alpha(n)$. Although this dependence on $n$ will turn out to be weak in the case when $\lim _{n \rightarrow \infty} \alpha(n)=\alpha^{*}$, we need to investigate carefully the dependence of the solution on $n$. This problem will appear first in Section 4, and in that section we shall indicate explicitly the dependence on the parameter $n$.

We shall prove under general conditions that (1.4) has a unique solution $(r, \bar{\varphi}) 0 \leq \bar{\varphi} \leq \pi$ which also satisfies relation (1.5). This result enables us to give a good asymptotic expression of (1.2) and to approximate $S^{(k)}(n)$ by a function of sum of independent random vectors. In such a way the limit behavior of $S^{(k)}(n)$ with an appropriately normalization can be described by means of a limit theorem for sums of independent random vectors. Since some technical conditions appear in the formulation of the results about the limit behavior of $S^{(k)}(n)$ we formulate them only in Section 2 .

The limit theorem for sums of independent random vectors needed in this paper may be interesting in itself. In this limit theorem, such a limit distribution appears whose coordinates are independent. This independence is not because of some uncorrelatedness property of the coordinates of the summands. It has a structural reason. It appears because the partial sums of such random vectors are considered whose first coordinates take values in a noncompact and the second coordinates in a compact space. (We consider such random vectors whose first coordinates, the absolute value of random complex numbers, take their values in the real line, and the second coordinates, the angle of these complex numbers, take their values in the unit circle.) Similar results in more general spaces were proved in [5]. 
This paper consists of six sections. In Section 2 we explain the method of the paper, formulate some technical results and the main theorems. In Section 3 we prove that under general conditions the asymptotic saddlepoint equation (1.4) together with relation (1.5) can be solved. In Section 4 we give a good asymptotic approximation of $S^{(k)}(n)$ by means of an expansion of the integrand in (1.2) around the solution of the saddlepoint equation (1.4). In Section 5 a limit theorem for sums of independent vectors needed in this paper is proved. Finally in Section 6 the main results of the paper are proved.

2. The strategy of the proof. Consider the function $H(r, \varphi)=H(z)$ defined in (1.3). First we want to prove that under general conditions for the distribution of $F(x)$ of the random variables $\xi_{j}(1.4)$ has a unique solution which also satisfies (1.5). In the proof we investigate the differentials of the function $H(r, \varphi)$. In these calculations the order of differentiation and expectation will be changed several times. To legitimate such steps some conditions will be imposed on the distribution of the distribution function $F(x)$.

It is simple to justify these calculations in the neighborhood of such points $z=r e^{i \varphi}$ for which the number $z$ has a nonzero imaginary part, that is, for which $\varphi \neq 0$ and $\varphi \neq \pi$. On the other hand, for $\varphi=0$ or $\varphi=\pi$ such a calculation is allowed only under fairly restrictive conditions. However, we shall differentiate only in the neighborhood of a point which can appear as the solution of (1.4) with some $\alpha(n)$; therefore we do not have to impose too-restrictive conditions. We shall formulate such a condition on $F(x)$ which probably can be weakened, but which is satisfied by all "nice" distribution functions. To formulate this condition let us introduce the functions

$$
K^{ \pm}(r)=E \frac{ \pm \xi}{(\xi \pm r)^{2}}, \quad r>0
$$

and sets

$$
\mathscr{A}^{ \pm}=\left\{r: r>0 \text { and } K^{ \pm}(r) \geq 0\right\},
$$

where $\xi$ is an $F(x)$-distributed random variable. Let us remark that the integral (2.1) is always meaningful, although the relation $E\left( \pm \xi /(\xi \pm r)^{2}\right)=-\infty$ is possible, since the integrands in these expressions have an upper bound depending only on $r$. As later calculation will show, it is enough to justify the change of order of expectation and differentiation only in a small neighborhood of the real numbers $r, r \in \mathscr{A}^{+} \cup \mathscr{A}^{-}$.

We formulate the following property.

Property A. If $r \in \mathscr{A}^{+}$, then there is a number $h=h(r)>0$ such that the interval $(-r-h,-r+h)$ has zero $F$ measure. If $r \in \mathscr{A}^{-}$, then there is a number $h=h(r)>0$ such that the interval $(r-h, r+h)$ has zero $F$ measure.

This property can be formulated in the following equivalent form. Let $\Sigma$ denote the support of the distribution of $\xi$, that is, the smallest closed set on the real line $R^{1}$ such that $P(\xi \in \Sigma)=1$. (Such a set exists. See, e.g., [4], 
Chapter 2, Theorem 2.1.) Then for all $r \in \mathscr{A}^{+} d(r,-\Sigma)>0$ and for all $r \in \mathscr{A}^{-}$ $d(r, \Sigma)>0$.

Property A is less restrictive than it may seem in the first moment, because the sets $\mathscr{L}^{ \pm}$are small. Thus for instance, $r \notin \mathscr{A}^{ \pm}$if the distribution function $F$ has a nonzero density function in a neighborhood of the point $\mp r$, or more generally if $F(\mp r+h)-F(\mp r)>C \eta^{2}$ or $F(\mp r)-F(\mp r-h)>C \eta^{2}$ with some $C>0, h>0$ and $0<\eta<h$. Indeed, $K^{ \pm}(r)=-\infty$ in this case. Thus Property A holds if for all $x F(x+h)-F(x-h) \geq$ const. $h^{2}$ or $F(x+h)-$ $F(x-h)=0$ if $h<h_{0}$. Here both $h_{0}$ and const. may depend on $x$. Let us also remark that also Property $\mathrm{A}$ holds if an $F$ distributed $\xi$ random variable is symmetrically distributed, since the sets $\mathscr{A}^{ \pm}$are empty in this case. Indeed, in this case,

$$
K^{ \pm}(r)=E \frac{ \pm \xi}{(r \pm \xi)^{2}}=\frac{1}{2} E\left(\frac{ \pm \xi}{(r \pm \xi)^{2}}+\frac{\mp \xi}{(r \mp \xi)^{2}}\right)=-E \frac{2 r \xi^{2}}{\left(\left(r^{2}-\xi^{2}\right)^{2}\right.}<0
$$

for all $r>0$.

We also assume that

$$
E|\xi|<\infty \text { and } E \frac{1}{|\xi|} I(\xi \neq 0)<\infty .
$$

We shall assume in the sequel that the distribution function $F$ satisfies Property A and (2.3). The following three lemmas which will be proved in Section 3 imply that if $P(\xi=0)<\alpha(n)<1$, then (1.4) has a unique solution which satisfies (1.5).

LEMMA 1. Fix some $r>0$ and consider the function $H(r, \varphi)$, defined in (1.3) as a function of $\varphi, 0 \leq \varphi \leq \pi$. [The function $H(r, \varphi)$ can also take the value $-\infty$ in the end points 0 and $\pi$.] The function $H$ has a unique maximum at a value $\bar{\varphi}=\bar{\varphi}(r)$ defined by

$$
\bar{\varphi}(r)= \begin{cases}0, & \text { if } E \frac{\xi}{(r+\xi)^{2}} \geq 0 \\ \pi, & \text { if } E \frac{\xi}{(r-\xi)^{2}} \leq 0 \\ \text { the unique solution of } & \\ \text { the equation } & \xi \\ E \frac{\xi}{r^{2}+\xi^{2}+2 r \xi \cos \varphi}=0 & \text { if } E \frac{\xi}{(r+\xi)^{2}}<0<E \frac{\xi}{(r-\xi)^{2}} .\end{cases}
$$

The relation

$$
\left.\frac{\partial H(r, \varphi)}{\partial \varphi}\right|_{\varphi=\bar{\varphi}}=0
$$

holds. 
Define the function $E(r, \varphi)=r(\partial / \partial r) H(r, \varphi)$ and $G(r)=E(r, \bar{\varphi}(r))$.

LEMMA 2. $G(r)$ is a continuous and strictly monotone increasing function.

Before the proof of Lemma 2 we prove the following technical Lemma A.

LEMMA A. The function $H(z)$ defined in (1.3) is analytic in the set $C \backslash(-\Sigma)$ and the functions $K^{ \pm}(z)$, the analytical continuation of the functions defined in (2.1), are analytic in the set $C \backslash(\mp \Sigma)$, where $C$ is the space of complex numbers, and $\Sigma$ is the support of the distribution of the random variable $\xi$. In particular, $K^{ \pm}(r)$ is continuous in the points $r \in \mathscr{A}^{ \pm}$. The numbers $r$ satisfying the equation $E\left(\xi /(\xi \pm r)^{2}\right)=0$ have no strictly positive condensation points.

\section{LEMMA 3.}

$$
\begin{aligned}
& \lim _{r \rightarrow \infty} G(r)=1, \\
& \lim _{r \rightarrow 0} G(r)=P(\xi=0)(=0 \text { if the distribution of } \xi \text { has no atom in } 0) .
\end{aligned}
$$

The second derivative of $H(r, \varphi)$ with respect to the variable $\varphi$ is nonpositive in the point $\bar{\varphi}(r)$, and it can be zero only if either $E\left(\xi /(r+\xi)^{2}\right)=0[$ in which case $\bar{\varphi}(r)=0]$ or if $E\left(\xi /(r-\xi)^{2}\right)=0$ [in which case $\left.\bar{\varphi}(r)=\pi\right]$. More explicitly,

$$
\begin{array}{r}
-2 E \frac{r^{2} \xi^{2} \sin ^{2} \varphi}{\left(r^{2}+\xi^{2}+2 r \xi \cos \varphi\right)^{2}}, \quad \text { if } 0<\bar{\varphi}(r)<\pi, \\
\left.\frac{\partial^{2}}{\partial \varphi^{2}} H(r, \varphi)\right|_{\varphi=\bar{\varphi}(r)}=-E \frac{r \xi}{(r+\xi)^{2}}\left(=-K^{+}(r)\right), \quad \text { if } \bar{\varphi}(r)=0, \\
E \frac{r \xi}{(r-\xi)^{2}}\left(=-K^{-}(r)\right), \quad \text { if } \bar{\varphi}(r)=\pi .
\end{array}
$$

The above relations imply that the saddlepoint equation (1.4) [together with property (1.5)] has a unique solution for $P(\xi=0)<\alpha(n)<1$, since a pair $(r, \varphi)$ is a solution if and only if $\varphi=\bar{\varphi}(r)$, where $\bar{\varphi}(r)$ is defined in Lemma 1 , and $G(r)=\alpha(n)$.

Let us rewrite formula (1.2) in the form

$$
S^{(k)}(n)=\Re\left(\frac{1}{\pi} \int_{0}^{\pi} \exp \left\{Z_{n}(r, \varphi)\right\} d \varphi\right)
$$

with

$$
Z_{n}(r, \varphi)=\sum_{j=1}^{n} \beta_{j}(r, \varphi)
$$


and

$$
\begin{aligned}
\beta_{j}(r, \varphi)= & \frac{1}{2} \log \left(r^{2}+\xi_{j}^{2}+2 r \xi_{j} \cos \varphi\right) \\
& +i \arccos \frac{r \cos \varphi+\xi_{j}}{\left(r^{2}+\xi_{j}^{2}+2 r \xi_{j} \cos \varphi\right)^{1 / 2}}-\alpha(n)(\log r+i \varphi),
\end{aligned}
$$

where $r$ is the first coordinate of the solution $(r, \bar{\varphi})$ of the fixed point equation (1.4) and (1.5). We shall give a good approximation of $S^{(k)}(n)$ in Section 4. To get it we impose the following

Property B. Let $(r, \bar{\varphi})=\left(r\left(\alpha^{*}\right)\right), \bar{\varphi}\left(r\left(\alpha^{*}\right)\right)$ be the solution of the fixed point equation (1.4) [together with relation (1.5)], if $\alpha(n)$ is replaced by $\alpha^{*}=$ $\lim _{n \rightarrow \infty} \alpha(n)$. Then

$$
E \frac{\xi}{(r \pm \xi)^{2}} \neq 0 \quad \text { for } r=r\left(\alpha^{*}\right)
$$

The integral in (2.8) can be well estimated. To do this we apply a Taylor expansion for $\beta_{j}(r, \varphi)$ in the variable $\varphi$ around the saddle point $\bar{\varphi}$ and then sum it up to get a good estimate for $Z_{n}(r, \varphi)$ defined in (2.9). The coefficients of this Taylor expansion are random. But since the random functions $\beta_{j}(r, \bar{\varphi})$ are independent, their sum can be well approximated, because of the laws of large numbers, by their expected values multiplied with $n$. The expected value of the first Taylor coefficient is zero because of (1.4). Indeed, the real part equals $\partial H(r, \varphi) / \partial \varphi=0$, and the imaginary part equals

$$
\frac{\partial}{\partial \varphi} E \arccos \frac{r \cos \varphi+\xi}{\left(r^{2}+\xi^{2}+2 r \xi \cos \varphi\right)^{1 / 2}}-\alpha(n)=r \frac{\partial H(r, \varphi)}{\partial r}-\alpha(n)=0
$$

in the point of solution $(r, \bar{\varphi})$ of (1.4). The identity (2.11) can be obtained by standard calculation. However, it is worth mentioning that this identity has a deeper reason. There are identities between the partial derivatives of the real part and analytic part of a complex analytic function, and the identity (2.11) expresses such properties formulated in a polar coordinate system.

By Lemma 3 the expected value of the second partial derivative of the real part of $\beta_{j}(r, \varphi)$ with respect to the variable $\varphi$ is nonpositive in the asymptotic saddlepoint $(r, \bar{\varphi}(r))$, and it is strictly negative if Property B holds. In this case the integral (2.8) is essentially concentrated in a small neighborhood of the point $\bar{\varphi}(r)$ with probability almost 1 (depending on $n$ ). In this small neighborhood of the point $\varphi(r)$, a small error is committed if all terms $\beta_{j}(r, \varphi)$ in (2.8) are replaced by their Taylor expansion around the point $\bar{\varphi}$ up to the second term. In such a way the integral in (2.8) can be approximated by a Gaussian integral which can be explicitly calculated. The above indicated calculation will be worked out in Section 4. Some additional technical difficulties arise if we want to show that the error term obtained in this calculation is negligible also if the real part of the integral in (2.8) is considered. To prove this fact we have to know that the integral in (2.8) with probability almost 
1 is such a complex number whose angle with the imaginary axis is not too small. We can prove this only under some additional restriction formulated a bit later. We introduce a condition which we shall call the stability of the level $\alpha^{*}=\lim _{n \rightarrow \infty} \alpha(n)$. In Proposition B of Section 5 we prove a limit theorem which helps us to overcome the above difficulties if the above-mentioned stability condition holds. The proofs in Section 5 are independent of the rest of the paper. The arguments formulated above lead to a result formulated in Lemma 4.

Before its formulation let us remark that, by the last statement of Lemma A, Property B is not a strong restriction. The exceptional set of the numbers $\alpha^{*}$ where it does not hold has no condensation points in the open interval $(P(\xi=0), 1)$. Moreover, in certain cases we know that this set is empty. This is the case for instance if $\xi$ has a symmetric distribution, since under this condition $\bar{\varphi}(r)=\pi / 2$ for all $r>0$. If Property B does not hold, then a more complicated picture arises. In this case not only the first but also the second derivative of the function $H(r, \varphi)-\alpha \log r$ disappears in the saddle point. Hence a more sophisticated method has to be applied and only weaker results can be obtained in this case. We shall not discuss this question in the present paper.

LEMMA 4. Let the distribution of $\xi$ satisfy Property A and (2.3). Beside this, let Property $B$ be satisfied with $r^{*}=\lim _{n \rightarrow \infty} r_{n}$, where $r_{n}$ is the solution of the asymptotic saddlepoint equation (1.4) [together with (1.5)] with the parameter $\alpha(n)$. Let us also assume that the level $\alpha^{*}=\lim _{n \rightarrow \infty} \alpha(n)$ is stable. (This notion will be introduced a bit later.) Put

$$
\bar{S}^{(k)}(n)=\left\{\begin{array}{cc}
\frac{\sqrt{2}}{\sqrt{K n \pi}} \exp \left\{n A_{0}+\sqrt{n} S_{0}-U_{1}\right\} \cos \left(n B_{0}+T_{0}-U_{2}-\frac{\omega}{2}\right), & \text { if } 0<\bar{\varphi}\left(r^{*}\right)<\pi, \\
=\frac{1}{\sqrt{2\left|A_{2}\right| \pi n}} \exp \left\{\frac{T_{1}^{2}}{2 A_{2}}+n A_{0}+\sqrt{n} S_{0}\right\}, & \text { if } \bar{\varphi}\left(r^{*}\right)=0, \\
=(-1)^{k(n)} \frac{1}{\sqrt{2\left|A_{2}\right| \pi n}} \exp \left\{\frac{T_{1}^{2}}{2 A_{2}}+n A_{0}+\sqrt{n} S_{0}\right\}, & \text { if } \bar{\varphi}\left(r^{*}\right)=\pi,
\end{array}\right.
$$

where the random variables $S_{0}=S_{0}(n), T_{0}=T_{0}(n), S_{1}=S_{1}(n), T_{1}=T_{1}(n)$ which are sums of independent random variables are defined in (4.8), (4.9) and (4.1), (4.2), the random variables $U_{1}=U_{1}(n), U_{2}=U_{2}(n)$ which are their transforms in (4.14). The constants $A_{0}=A_{0}(n), B_{0}=B_{0}(n), A_{2}=A_{2}(n), K$ and $\omega=\omega(n)$ are defined in (4.3), (4.4) and (4.14'). Then

$$
\frac{S^{(k)}(n)}{\bar{S}^{(k)}(n)} \Rightarrow 1,
$$

where $\Rightarrow$ denotes convergence in probability. 
Lemma 4 plays a crucial role in our investigation, because it enables us to replace the expression $S^{(k)}(n)$ introduced in (1.1) by $\bar{S}^{(k)}(n)$ defined in (2.12) when we are interested in its limit behavior. The expression $\bar{S}^{(k)}(n)$ is a functional of the random variables $S_{0}(n), S_{1}(n), T_{0}(n)$ and $T_{1}(n)$ which are normalized sums of independent random variables. The asymptotic behavior of $S_{0}(n), S_{1}(n)$ and $T_{1}(n)$ is described by the central limit theorem while that of $T_{0}(n)$ by limit theorems for sums of independent random variables on the compact group $[0,1] \bmod 1$, where the group action is summation modulo 1. However, these classical results are not sufficient for our purposes, we also want to control the limit of the joint distribution of the above random variables. Hence we formulate the following Proposition A whose proof will be given in Section 5. It implies that $T_{0}(n)$ is asymptotically independent from the other partial sums, because it takes values on a compact group, while the other partial sums take values on a noncompact group. Before formulating this result we introduce some notations and make some remarks.

We shall identify the group $G=[0,1)$ with summation modulo 1 with the unit circle. Let us remark that the closed subgroups $G_{0}$ of $G$ are the group $G$ itself and the discrete groups of the form $G_{0}=\{j / p ; j=0, \ldots, p-1\}$ with some positive integer $p$. A coset of a finite subgroup $G_{0}$ is of the form $G_{0}+\alpha$ with some $0 \leq \alpha<1$. For all probability measures $\mu$ on $(0,1]$, there is a smallest closed set, called the support of the measure, whose $\mu$ measure is 1 . For all probability measures $\mu$ there is a minimal coset $G_{0}+\alpha$ which contains the support of $\mu$. This means that the $\mu$ measure of this coset is 1 , and all cosets with this property contain this coset. If no coset of a finite subgroup of $G$ has this property, then we call the whole group $G$ the minimal coset which contains the support of the measure $\mu$. Now we formulate the following.

Proposition A. Let $\left(X_{n}, Y_{n}\right), n=1,2, \ldots$, be a sequence of i.i.d. random vectors such that $X_{n}$ is a random vector in $R^{k}$ with expectation zero and covariance matrix $\Sigma, Y_{n}$ is a random variable on the unit circle $G=[0,1)$. Let $G_{0}+\alpha$ be the minimal coset which contains the support of the distribution of $Y_{n}$. Put $U_{n}=(1 / \sqrt{n}) \sum_{s=1}^{n} X_{s}, V_{n}=\sum_{s=1}^{n} Y_{s}-n \alpha$. Then the joint distribution of $\left(U_{n}, V_{n}\right)$ tends to the distribution of a random vector $(U, V)$, where $U$ has normal distribution with expectation zero and covariance $\Sigma, V$ is uniformly distributed on the subgroup $G_{0}$ of $G$, and the random variables $U$ and $V$ are independent. In the case $G_{0}=G, \alpha$ can be chosen in an arbitrary way, for example, $\alpha=0$.

The result of Proposition A is not sufficient in itself for our purposes. The reason for this is that the distributions of the random variables we are investigating depend on a parameter $\alpha(n)$. This parameter satisfies the relation $\alpha(n) \rightarrow \alpha^{*}$, but it may depend on $n$. Hence we need such a version of Proposition A where the distribution of the random variables $X_{j}=X_{j}(n)$ and $Y_{j}=Y_{j}(n), j=1, \ldots, n$, may weakly depend on $n$. Let us remark that, in the limit theorems for sums of independent random variables on a compact group $G$, no normalization is taken; hence even a small perturbation of the 
summands may radically change the limit distribution of their sums. Nevertheless, we show that in the case when the distribution of $Y_{n}$ is close to a measure which is not concentrated in a coset of a closed finite subgroup, a version of Proposition A can be proved where the distribution of the summands may depend on $n$. To formulate this result first we introduce the following definition.

Definition. We call a probability measure $\mu$ on the group $G=[0,1)$, $\bmod 1$ stable if for all finite cosets $K=\{j / p+c, j=0, \ldots, p-1\}$, with a positive integer $p$ and $0 \leq c<1, \mu(K)<1$, or in other words, the minimal coset which contains the support of the measure $\mu$ is the whole group $G$.

This terminology for stable distribution differs from the traditional one, but since we apply it on a different space, hopefully it causes no confusion. Now we formulate the following result.

Proposition 2. For all $n$ let $\left(X_{j}(n), Y_{j}(n)\right), j=1,2, \ldots, n$, be a sequence of i.i.d. random vectors with the following properties: $X_{j}(n)$ are i.i.d. random vectors in $R^{k}, E X_{j}(n)=0$, the relation $E\left\|X_{1}(n)-X\right\|^{2} \rightarrow 0$ holds with a random variable $X$ in $R^{k}, E X=0$, which has a covariance matrix $\Sigma, Y_{j}(n)$ is a random variable on the unit circle $[0,1)$ with a distribution $\mu_{n}$ on $[0,1)$ such that $\mu_{n} \Rightarrow \mu$ and $\mu$ is a stable probability measure on $[0,1)$, where $\Rightarrow$ denotes weak convergence of measures. Define the random variables $U_{n}=(1 / \sqrt{n}) \sum_{s=1}^{n} X_{s}(n)$ and $V_{n}=\sum_{s=1}^{n} Y_{s}(n) \bmod 1$. Then the joint distribution of $\left(U_{n}, V_{n}\right)$ tends to the distribution of a random vector $(U, V)$, where $U$ has normal distribution with expectation zero and covariance $\Sigma, V$ is uniformly distributed on $G=[0,1)$ and the random variables $U$ and $V$ are independent.

Propositions A and B hold because one of the coordinates of the random vectors we are summing up takes value in a compact group while the other component takes value in a noncompact group. Results similar to Proposition A can be found in [5] in a more general setting, but to find the right generalization of Proposition B seems to be an interesting open question.

The above results enable us to investigate the limit behavior of the random variable $S^{(k)}(n)$ defined in (1.1). But because of the conditions we had to impose in the limit theorem formulated in Proposition B, we can prove these results only under certain restrictions. Let us introduce the following terminology.

Definition. We call the level $\alpha^{*}$ stable if one of the following conditions are satisfied:

(i) Either $E\left( \pm \xi /(r \pm \xi)^{2}\right)>0$ for $r=r\left(\alpha^{*}\right)$, that is, either $\bar{\varphi}(\alpha)=0$ or $\bar{\varphi}(\alpha)=\pi$ if $\alpha$ is in a small neighborhood of $\alpha^{*}$; 
(ii) or $0<\bar{\varphi}\left(\alpha^{*}\right)<\pi$, and the distribution of the random variable,

$$
Y=\frac{1}{2 \pi} \arccos \frac{r \cos \bar{\varphi}+\xi}{\left(r^{2}+\xi^{2}+2 r \xi \cos \bar{\varphi}\right)^{1 / 2}},
$$

where $r=r\left(\alpha^{*}\right)$ and $\bar{\varphi}=\bar{\varphi}\left(\alpha^{*}\right)$ is a stable distribution on the unit circle $[0,1)$.

We can give a good asymptotic of the symmetric statistics $S^{(k)}(n)$ if $(n-k) / n=\alpha(n) \rightarrow \alpha^{*}$ with a stable level $\alpha^{*}$.

If $0<\bar{\varphi}\left(\alpha^{*}\right)<\pi$, then the second condition of the stability of $\alpha^{*}$ holds in the generic case, but the description of the exceptional numbers $\alpha^{*}$ and distributions $F$ seems to be a hard number theoretic problem. Now we formulate the following theorem.

Theorem 1. Let Property A and relation (2.3) hold, and let $\alpha^{*}$ be a stable level. If $\alpha(n)=(n-k) / n \rightarrow \alpha^{*}$ as $n \rightarrow \infty$, then the random variables

$$
\frac{\log \left|S^{(k)}(n)\right|-n A_{0}(n)}{\sqrt{n}}
$$

[with $S^{(k)}(n)$ defined in (1.1)] converge in distribution to the normal law with expectation zero and variance Var $\eta$, where $\eta=\eta(\bar{\varphi})=\frac{1}{2} \log \left(r\left(\alpha^{*}\right)^{2}+\xi^{2}+\right.$ $\left.2 r\left(\alpha^{*}\right) \xi \cos \bar{\varphi}\left(\alpha^{*}\right)\right),\left(r\left(\alpha^{*}\right), \varphi\left(\alpha^{*}\right)\right)$ is the solution of the saddlepoint equation (1.4) if the number $\alpha(n)$ is replaced by $\alpha^{*}=\lim _{n \rightarrow \infty} \alpha(n)$, and $A_{0}=A_{0}(n)$ is defined in (4.3). $\left|S^{(k)}(n)\right|$ can be replaced by $S^{(k)}(n)$ in the case $\bar{\varphi}\left(\alpha^{*}\right)=0$, by $(-1)^{k} S^{(k)}(n)$ in the case $\bar{\varphi}\left(\alpha^{*}\right)=\pi$ in (2.13), while in the case $0<\bar{\varphi}\left(\alpha^{*}\right)<\pi$ $P\left(\operatorname{sign} S^{(k)}(n) \rightarrow 1\right)=1 / 2$ and $\log \left|S^{(k)}(n)\right|$ and $\operatorname{sign} S^{(k)}(n)$ are asymptotically independent.

Theorem 1 does not contain the result of [3], where the limit theorem is given for a normalized version of $S^{(k)}(n)$ (without logarithm) if the random variables $\xi_{j}$ have the distribution $P\left(\xi_{j}=1\right)=P\left(\xi_{j}=-1\right)=1 / 2$. In this case the random variable $\eta$ is constant, $\operatorname{Var} \eta=0$, and the limit (2.13) is degenerate. In the following Lemma 5 we describe those distributions $F$ and levels $\alpha^{*}$ for which the limit distribution in Theorem 1 is degenerate. Then we shall describe the limit behavior of $S_{n}^{(k)}$ in such cases.

LEMMA 5. The random variable $\eta=\eta\left(\alpha^{*}\right)=\frac{1}{2} \log \left(r\left(\alpha^{*}\right)^{2}+\xi^{2}+2 r \xi \cos \bar{\varphi}\left(\alpha^{*}\right)\right)$ appearing in Theorem 1 is constant, if an $F$ distributed random variable $\xi$ is concentrated in two points, that is, there are two numbers $x_{1}, x_{2}$ such that $P\left(\xi=x_{1}\right)=p, P\left(\xi=x_{2}\right)=q=1-p$, and one of the following conditions is satisfied:

(a) $0<\bar{\varphi}\left(\alpha^{*}\right)<\pi$, in which case $E \xi=p x_{1}+q x_{2}=0, \alpha^{*}>1-4 p q$.

(b) $\bar{\varphi}\left(\alpha^{*}\right)=0$, in which case $\alpha^{*}=-\left((p-q)\left(x_{1}+x_{2}\right) / x_{1}-x_{2}\right), E \xi=p x_{1}+$ $q x_{2} \geq 0$ and $x_{1}+x_{2}<0$.

(c) $\bar{\varphi}\left(\alpha^{*}\right)=\pi$, in which case $\alpha^{*}=-\left((p-q)\left(x_{1}+x_{2}\right) / x_{1}-x_{2}\right), E \xi=p x_{1}+$ $q x_{2} \leq 0$ and $x_{1}+x_{2}>0$. 
In Theorem 2 we describe the limit behavior of $S^{(k)}(n)$ in case (a) of Lemma 5. It contains the result of [3].

THEOREM 2. Let the distribution of the random variable $\xi$ have the form $P\left(\xi=x_{1}\right)=p, P\left(\xi=x_{2}\right)=q=1-p, p x_{1}+q x_{2}=0$, that is, $E \xi=0$. Let $(n-k) / n=\alpha(n) \rightarrow \alpha^{*}$ with some stable level $\alpha^{*}$ such that $1>\alpha^{*}>1-4 p q$.

Then the random variables

$$
\frac{\sqrt{K \pi n}}{\sqrt{2}} \exp \left(-A_{0}(n)\right) S^{(k)}(n)
$$

converge in distribution to the random variable

$$
\exp \left\{\frac{A_{2}\left(S^{2}-T^{2}\right)+2 B_{2} S T}{2\left(A_{2}^{2}+B_{2}^{2}\right)}\right\} \cos Z
$$

as $n \rightarrow \infty$, where the constants $A_{0}, A_{2}, B_{2}$ and $K$ are defined in (4.3), (4.4), $\left(4.14^{\prime}\right)$, more precisely, they are the limits of these quantities depending on $n$ as $n \rightarrow \infty,(S, T)$ is a Gaussian random vector with expectation zero, $Z$ is a random variable, uniformly distributed in $[0,2 \pi)$ and independent of the vector $(S, T)$, and

$$
\begin{aligned}
E S^{2} & =\operatorname{Var} \frac{-r \xi \sin \bar{\varphi}}{r^{2}+\xi^{2}+2 r \xi \cos \bar{\varphi}}, \\
E T^{2} & =\operatorname{Var} \frac{r \xi \cos \bar{\varphi}+r^{2}}{r^{2}+\xi^{2}+2 r \xi \cos \bar{\varphi}}, \\
\operatorname{Cov}(S, T) & =\operatorname{Cov}\left(\frac{-r \xi \sin \bar{\varphi}}{r^{2}+\xi^{2}+2 r \xi \cos \bar{\varphi}}, \frac{r \xi \cos \bar{\varphi}+r^{2}}{r^{2}+\xi^{2}+2 r \xi \cos \bar{\varphi}}\right),
\end{aligned}
$$

where $r=r\left(\alpha^{*}\right), \bar{\varphi}=\bar{\varphi}\left(\alpha^{*}\right)$.

Finally, in Theorem $2^{\prime}$ we describe the limit behavior of $S^{(k)}(n)$ in the case when the conditions of part (b) of Lemma 5 hold. The case when the conditions of part (c) hold can be obtained by applying this result for the random variables $-\xi_{j}$ which satisfy part (b).

THEOREM 2'. Let the distribution of $\xi$ satisfy the following conditions: $P\left(\xi=x_{1}\right)=p, P\left(\xi=x_{2}\right)=q=1-p$ with some $x_{1}, x_{2}$ and $p$ such that $p x_{1}+q x_{2}>0$ and $x_{1}+x_{2}<0, x_{1}>x_{2}$. Put

$$
\alpha^{*}=\frac{(p-q)\left(-x_{1}-x_{2}\right)}{x_{1}-x_{2}} .
$$


If $(n-k) / n=\alpha(n) \rightarrow \alpha^{*}$, then the symmetric polynomial $S^{(k)}(n)$ satisfies the following limit theorem:

$$
\begin{aligned}
& \exp \left\{\frac{T^{2}}{A_{2}}\right\}, \quad \text { if } \sqrt{n}\left(\alpha(n)-\alpha^{*}\right) \rightarrow 0, \\
& \exp \left\{\frac{T^{2}}{A_{2}}+c L V\right\}, \\
& \quad \text { if } \sqrt{n}\left(\alpha(n)-\alpha^{*}\right) \rightarrow c, 0<|c|<\infty,
\end{aligned}
$$

where $L=\left(\sqrt{p q}\left(x_{1}-x_{2}\right) / p x_{1}+q x_{2}\right), T=-\left(x_{1}+x_{2} / x_{1}-x_{2}\right) V$, and $V$ is a standard normal random variable.

If $\left|\sqrt{n}\left(\alpha(n)-\alpha^{*}\right)\right| \rightarrow \infty$, there is not such a natural scaling of $S^{(k)}(n)$ as in the previous cases.

3. The solution of the fixed point equation. In this section we prove Lemmas 1, 2 and 3 which imply that there is a unique solution of (1.4), $0 \leq$ $\varphi \leq \pi$, which also satisfies relation (1.5).

Proof of Lemma 1. Let us define the function $L(r, \psi)=\frac{1}{2} E \log \left(r^{2}+\xi^{2}+\right.$ $2 r \xi \psi),-1 \leq \psi \leq 1$. This function is obtained if $\psi$ is written instead of $\cos \varphi$ in the function $H(r, \varphi)$. It is a concave function of the variable $\psi$ in the open interval $-1<\psi<1$ for all $r>0$, since its second derivative is negative. The behavior of the function $L(r, \psi)$ in the end point $\psi=1$ can be investigated by means of the following observation. There is a sufficiently small $\varepsilon>0$ such that in the interval $1-\varepsilon<\psi<1$ either $L(r, \psi)$ is monotone decreasing and the derivative $\partial L(r, \psi) / \partial \psi$ is negative or $L(r, \psi)$ is monotone increasing and the derivative $\partial L(r, \psi) / \partial \psi$ is positive. In the first case,

$$
\begin{aligned}
L(r, 1)-L(r, \psi) & =\frac{1}{2} E \log \left(1+\frac{2(1-\psi) r \xi}{r^{2}+\xi^{2}+2 r \xi \psi}\right) \\
& \leq E \frac{(1-\psi) r \xi}{r^{2}+\xi^{2}+2 r \xi \psi}=(1-\psi) \frac{\partial L}{\partial \psi}<0,
\end{aligned}
$$

and $L(r, 1)<\sup L(r, \psi)$.

In the second case it follows from (2.3) and Fatou's lemma that

$$
0 \leq \limsup _{\psi \rightarrow 1} \frac{\partial L}{\partial \psi}=\limsup _{\psi \rightarrow 1} E \frac{r \xi}{r^{2}+\xi^{2}+2 r \xi \psi} \leq E \frac{r \xi}{(r+\xi)^{2}}=r K^{+}(r),
$$

where the function $K^{+}(r)$ is defined in (2.1). Hence $r \in \mathscr{L}^{+}$, and Property A can be applied. This implies in particular that $L(r, 1)=\lim _{\psi \rightarrow 1} L(r, \psi)=$ $\sup _{0 \leq \psi \leq 1} L(r, \psi)$. Similarly, $\psi=-1$ is the maximum of $L(r, \psi)$ if and only if the function $L(r, \psi)$ is monotone decreasing in the interval $(-1,-1+\varepsilon)$ with 
a sufficiently small $\varepsilon>0$, and $r \in \mathscr{A}^{-}$, that is, $K^{-}(r) \geq 0$. In particular, the function $L(r, \psi)$ is continuous in the point $\psi=-1$ in this case.

The above results imply that the function $H(r, \varphi)$ has a unique maximum in the interval $0 \leq \varphi \leq \pi$. The maximum is in the point $\varphi=0$ if the function $L(r, \psi)$ has its maximum at $\psi=1$ which holds if $K^{+}(r) \geq 0$. It has its maximum at $\varphi=\pi$ if $L(r, \psi)$ has its maximum at $\psi=-1$ and $K^{-}(r) \geq 0$. These statements are equivalent to the first two lines of (2.4). The maximum is in the open interval $0<\varphi<\pi$ if $-K^{-}(r)<0<K^{+}(r)$. In this case $\partial H(r, \varphi) / \partial \varphi=0$ in the place of maximum, and since the order of differentiation and expectation can be changed, this fact implies the third line of (2.4). Finally, relation (2.5) also holds for $\bar{\varphi}=0$ and $\bar{\varphi}=\pi$. To see this, observe that since $r \in \mathscr{A}^{-}$if $\bar{\varphi}=0, r \in \mathscr{A}^{+}$if $\bar{\varphi}=\pi, \partial H(r, \varphi) / \partial \varphi=0$ in the place of maximum $\bar{\varphi}$, and the order of differentiation and expectation can be changed in this case, too.

Let us introduce the notation $U=U(r, \xi, \varphi)=r^{2}+\xi^{2}+2 r \xi \cos \varphi$. Now we turn to the following proof.

Proof of Lemma A. If $z_{0}=r_{0} \exp \left(i \varphi_{0}\right) \notin-\Sigma$, and $\xi \in \Sigma$, then for all $z=r e^{i \varphi}$ in a sufficiently small neighborhood of $z_{0}$ the number $|z+\xi|^{2}=$ $U(r, \xi, \varphi) \geq C>0$ with an appropriate number $C=C\left(z_{0}\right)$. Hence the function $\log U(r, \xi, \varphi)$ is analytic in such a small neighborhood of $z_{0}$, and it is separated from $-\infty$ (independently of $\xi \in \Sigma$ ). Then, since $\log U(r, \xi, \varphi) \leq$ const. $(|\xi|+r)$, and relation (2.3) holds, we get by taking expectation that $H(z)=\frac{1}{2} E \log U(r, \xi, \varphi)$ is analytic in a small neighborhood of $z_{0}$.

Similarly, if $z_{0} \notin \mp \Sigma, \xi \in \Sigma$ and $z$ is in a small neighborhood of $z_{0}$, then $\left|\xi /(\xi \pm z)^{2}\right| \leq C<\infty$, and taking expectation we get that the functions $K^{ \pm}(z)$ are analytic in the domain $C \backslash(\mp \Sigma)$. In particular, Property A implies that the function $K^{ \pm}(r)$ is continuous in the points $r \in \mathscr{A}^{ \pm}$.

Moreover, the function $K^{ \pm}(r)$ defined for all $r>0$ is upper semicontinuous, hence the sets $\mathscr{A}^{ \pm}$defined in (2.2) are closed subsets of the positive numbers. We show that there is no sequence $r_{n}, n=1,2, \ldots$, with a limit $0<r=$ $\lim _{n \rightarrow \infty} r_{n}<\infty$ such that $K^{ \pm}\left(r_{n}\right)=0$ for all $n$. Indeed, the limit $r$ would be also in the set $\mathscr{A}^{ \pm}$, and because of Property A, the relation $d(r, \mp \Sigma)>0$ would hold. This would imply that $K^{ \pm}(z) \equiv 0$ in the domain of analyticity of the function $K^{ \pm}(z)$. This relation also would imply that $E(\xi / \xi \pm z)=0$ on the set $\Im z>0$, since the derivative of this function is $K^{ \pm}(z) \equiv 0$, and as a consequence it is a constant function. Then choosing $z=i u, u \rightarrow \infty$ we get that this constant is zero. On the other hand, we get with the choice $z=i u$, $u \rightarrow 0$ that this constant is $P(\xi \neq 0) \neq 0$, and this is a contradiction.

Now we turn to the proof.

ProOF OF LEMMA 2. We shall prove that

$$
\begin{aligned}
\frac{d G(r)}{d r}>0 \text { if } E \frac{\xi}{(r+\xi)^{2}}< & 0<E \frac{\xi}{(r-\xi)^{2}} \\
& \text { (or equivalently, if } 0<\bar{\varphi}(r)<\pi),
\end{aligned}
$$


and also

$$
\frac{d G(r)}{d r}>0 \quad \text { if } \quad E \frac{\xi}{(r+\xi)^{2}}>0 \quad \text { or } \quad E \frac{\xi}{(r-\xi)^{2}}<0 .
$$

Finally, we show that the function $G(r)$ is continuous for all $r>0$. This continuity, the last statement of Lemma A, together with (3.1) and (3.2) imply that in an interval $[a, b], 0<a<b<\infty, G(r) / d r>0$ with the possible exception only of finitely many points. Lemma 2 follows from this fact.

To prove relation (3.1) observe that in this case $E\left(\xi / r^{2}+\xi^{2}+2 r \xi \cos \bar{\varphi}(r)\right)=$ 0 . This identity determines the function $\bar{\varphi}(r)$ in the small neighborhood of a point $(r, \bar{\varphi}(r))$. The implicit function theorem enables us to calculate the function $\bar{\varphi}^{\prime}(r)$. We get that

$$
\bar{\varphi}^{\prime}(r)=\frac{E\left(2 \xi(r+\xi \cos \bar{\varphi}) / U^{2}\right)}{E\left(2 \xi^{2} r \sin \bar{\varphi} / U^{2}\right)}=\frac{\cos \bar{\varphi}}{r \sin \bar{\varphi}}+\frac{E\left(\xi / U^{2}\right)}{\sin \bar{\varphi} E\left(\xi^{2} / U^{2}\right)} .
$$

Exploiting again that the third line of (2.4) holds in this case, we get that

$$
\begin{aligned}
G(r)=E(r, \bar{\varphi}(r)) & =E \frac{r^{2}+r \xi \cos \bar{\varphi}(r)}{U(r, \xi, \bar{\varphi}(r))} \\
& =E \frac{r^{2}}{U(r, \xi, \bar{\varphi}(r))}=1-E \frac{\xi^{2}}{U(r, \xi, \bar{\varphi}(r))}
\end{aligned}
$$

and

$$
\begin{aligned}
\frac{d G(r)}{d r} & =E \frac{2 \xi^{2}(r+\xi \cos \bar{\varphi}(r))}{U^{2}(r, \xi, \bar{\varphi}(r))}-\bar{\varphi}^{\prime}(r) E \frac{2 r \xi^{3} \sin \bar{\varphi}(r)}{U^{2}(r, \xi, \bar{\varphi}(r))} \\
& =E \frac{2 r \xi^{2}}{U^{2}(r, \xi, \bar{\varphi}(r))}-\frac{E\left(\xi / U^{2}(r, \xi, \bar{\varphi}(r))\right) E\left(2 r \xi^{3} / U^{2}(r, \xi, \bar{\varphi}(r))\right)}{E\left(\xi^{2} / U^{2}(r, \xi, \bar{\varphi}(r))\right)}
\end{aligned}
$$

if $0<\bar{\varphi}(r)<\pi$. Hence relation (3.1) is equivalent to the inequality

$$
E \frac{r^{2} \xi}{U^{2}} E \frac{\xi^{3}}{U^{2}}<\left(E \frac{r \xi^{2}}{U^{2}}\right)^{2}
$$

or since the third line in (2.4) implies that

$$
E \frac{r \xi^{2}}{U^{2}}=\frac{1}{2 \cos \bar{\varphi}} E \frac{2 r \xi^{2} \cos \bar{\varphi}-\xi U}{U^{2}}=-\frac{1}{2 \cos \bar{\varphi}}\left(E \frac{\xi^{3}}{U^{2}}+E \frac{r^{2} \xi}{U^{2}}\right),
$$

it is also equivalent to the inequality

$$
\left(4 \cos ^{2} \bar{\varphi}-2\right) E \frac{\xi^{3}}{U^{2}} E \frac{r^{2} \xi}{U^{2}}<\left(E \frac{\xi^{3}}{U^{2}}\right)^{2}+\left(E \frac{r^{2} \xi}{U^{2}}\right)^{2} .
$$

The Cauchy-Schwarz inequality implies that the last inequality and hence relation (3.1) holds. To see that this formula holds with a strict inequality it is enough to observe that $\left|4 \cos ^{2} \bar{\varphi}-2\right|<2$ for $0<\bar{\varphi}<\pi$, and the equations $E\left(\xi^{3} / U^{2}\right)=0$ and $E\left(r^{2} \xi / U^{2}\right)=0$ cannot hold simultaneously. Indeed, they 
would imply together with the third line of (1.4) for $r>0$ and $0<\bar{\varphi}<\pi$ that $E\left(\xi^{2} / U^{2}\right)=0$, and this is impossible.

To prove relation (3.2) let us observe that if $E\left(\xi /(r+\xi)^{2}\right)>0$, then $\bar{\varphi}(r)=$ 0 , and because of Property A the order of differentiation with respect to the variable $r$ and expectation can be changed when $G(r)$ and $d G(r) / d r$ are calculated. Simple calculation shows that $G(r)=E(r, \bar{\varphi}(r))=E(r / \xi+r)$, $d G(r) / d r=E\left(\xi /(r+\xi)^{2}\right)>0$, and if $E\left(\xi /(r-\xi)^{2}\right)<0$, then $\bar{\varphi}(r)=\pi$, and $d G(r) / d r=-E\left(\xi /(r-\xi)^{2}\right)>0$. These formulas imply (3.2).

The above arguments also show the continuity of the function $G(r)$ except the points $r$ such that $E\left(\xi /(\xi \pm r)^{2}\right)=0$. To prove the continuity in these points it is enough to show that the function $\bar{\varphi}(r)$ defined in Lemma 1 is continuous in these points. To prove this observe that in these points either $\bar{\varphi}(r)=0$ or $\bar{\varphi}(r)=\pi$. If $\bar{\varphi}(r)=0$, then, as we showed in the proof of Lemma 1 , the expression in the third line of (2.4) is strictly negative for this $r$ and $0<\varphi \leq \pi$. This function is uniformly continuous (analytic) and separated from zero in a small neighborhood of the set $\left\{z: z=r e^{i \varphi}\right\}$, with this $r$ and $\varepsilon \leq \varphi \leq \pi$ for arbitrary $\varepsilon>0$. This implies that $\bar{\varphi}(r)$ is continuous in this exceptional set if $\bar{\varphi}=0$. The case $\bar{\varphi}=\pi$ can be handled similarly. Lemma 2 is proved.

Proof of LemMa 3. Since $G(r)$ is a monotone increasing function it is enough to prove the formulas in relation (2.6) for a special sequence $r_{n} \rightarrow \infty$ and $r_{n} \rightarrow 0$. To prove the first relation, let us first consider the case when there is a sequence of numbers $r_{n} \rightarrow \infty$ such that $0<\bar{\varphi}\left(r_{n}\right)<\pi$. By relation (3.4), Fatou's lemma and the observation $r_{n}^{2} / U \rightarrow 1, \xi^{2} / U \rightarrow 0, \xi^{2} / U \geq 0$, $r^{2} / U \geq 0$ imply that

$$
\begin{aligned}
& \liminf _{r \rightarrow \infty} G(r)=\liminf _{r \rightarrow \infty} E \frac{r^{2}}{U} \geq 1, \\
& \limsup _{r \rightarrow \infty} G(r)=1-\liminf _{r \rightarrow \infty} E \frac{\xi^{2}}{U} \leq 1,
\end{aligned}
$$

hence the first line of relation of (2.6) holds in this case. Similarly if $r_{n} \rightarrow 0$, $0<\bar{\varphi}\left(r_{n}\right)<\pi$, then $r^{2} / U \rightarrow I(\xi=0)$ and $\xi^{2} / U \rightarrow 1-I(\xi=0)$. Then a similar argument proves the second line of (2.6) in this case.

In the remaining cases, we have, because of the continuity of the function $\bar{\varphi}(r)$, either $\bar{\varphi}(r)=0$ and $E\left(\xi /(\xi+r)^{2}\right) \geq 0$ or $\bar{\varphi}(r)=\pi$ and $E\left(-\xi /(\xi-r)^{2}\right) \geq$ 0 for all $r \geq r_{0}$ with some $r_{0}>1$ if the case $r \rightarrow \infty$ is considered. We claim that $-\Sigma \cap\left\{r: r>r_{0}\right\}$ is empty for $r>r_{0}$ in the first case, and $\Sigma \cap\left\{r: r>r_{0}\right\}$ is empty for $r>r_{0}$ is empty in the second case, where $\Sigma$ denotes the support of the distribution of the $\xi$. Indeed, if this relation did not hold, then in the first case one could find by a halving procedure a sequence of intervals $\left[a_{n}, b_{n}\right]$ such that $b_{n}>a_{n}>r_{0}, b_{n}-a_{n}=2^{-n}, F\left(-b_{n}\right)-F\left(-a_{n}\right) \geq K 2^{-n}$ with some appropriate $K>0$ for all $n=1,2, \ldots$, where $F$ is the distribution function of the random variable $\xi$. Let $R$ be the intersection of the intervals $\left[a_{n}, b_{n}\right]$, 
$n=1,2, \ldots$ Then $R>r_{0}$, and we claim that $E\left(\xi /(\xi+R)^{2}\right)=-\infty$ which is a contradiction. This equation holds, because for all $n>0$,

$$
E \frac{\xi}{(\xi+R)^{2}} \leq \int_{-b_{n}}^{-a_{n}} \frac{x}{(R+x)^{2}} F(d x)+E \xi I(\xi \geq 0) \leq- \text { const. } 2^{n}+\text { const. },
$$

and we get the above relation as $n \rightarrow \infty$. The proof in the case $\bar{\varphi}=\pi$ for $r \geq r_{0}$ is similar.

It follows from the above proved statement, the relation $\lim _{r \rightarrow \infty}(r / r \pm \xi)=1$ with probability 1 and the Lebesgue convergence theorem that $\lim _{r \rightarrow \infty} G(r)=$ $\lim _{r \rightarrow \infty} E(r / r \pm \xi)=1$ in this case too.

The limit behavior in the case $r \rightarrow 0$ can be handled similarly. If there is no sequence $r_{n} \rightarrow 0$ such that $0<\bar{\varphi}\left(r_{n}\right)<\pi$, then there is a number $1>r_{0}>0$ such that either $\bar{\varphi}(r)=0$ or $\bar{\varphi}(r)=\pi$ for all $0<r<r_{0}$. In the first case $-\Sigma \cap\left\{r: 0<r<r_{0}\right\}=\varnothing$, and in the second case $\Sigma \cap\left\{r: 0<r<r_{0}\right\}=\varnothing$. This can be proved similarly to the case $r \rightarrow \infty$ with an estimate similar to (3.5) with the difference that in this case the relation $E\left(\xi /(R+\xi)^{2}\right) I(\xi>0) \leq$ $E\left(\xi / \xi^{2}\right) I(\xi>0) \leq E(I(\xi \neq 0) /|\xi|)<\infty$ holds.

Finally, as $\lim _{r \rightarrow 0}(r / r \pm \xi)=I(\xi=0)$, the Lebesgue dominated convergence theorem implies that $\lim _{r \rightarrow 0} G(r)=\lim _{r \rightarrow 0} E(r / r \pm \xi)=E I(\xi=0)$. Relation (2.6) is proved.

We have proved that the saddlepoint equation (1.4) and (1.5) has a unique solution if $P(\xi=0)<\alpha(n)<1$. Let us calculate the second partial derivative of $F(r, \bar{\varphi})$ with respect to the variable $\varphi$ in the saddlepoint. We get that

$$
\frac{\partial^{2}}{\partial \varphi^{2}} H(r, \varphi)=-E \frac{r \xi \cos \varphi}{U}-2 E \frac{r^{2} \xi^{2} \sin ^{2} \varphi}{U^{2}}
$$

in a general point $(r, \varphi)$. Then a simple substitution implies (2.7). Lemma 3 is proved.

4. Asymptotic approximation for the symmetric polynomial $S^{(k)}(n)$. Let us consider the solution $\left(r_{n}, \bar{\varphi}_{n}\right)$ of the asymptotic saddlepoint equation (1.4) which also satisfies relation (1.5). Let us remark that these numbers depend on $n$ because of the function $\alpha(n)$ at the right-hand side of (1.4). On the other hand, if $\left(r\left(\alpha^{*}\right), \bar{\varphi}\left(\alpha^{*}\right)\right)$ denotes the solution of (1.4) with the modification that the number $\alpha(n)$ is replaced by $\alpha^{*}=\lim _{n \rightarrow \infty} \alpha(n)$ in it, then $\lim _{n \rightarrow \infty} r_{n}=r\left(\alpha^{*}\right)$, and $\lim _{n \rightarrow \infty} \bar{\varphi}_{n}=\bar{\varphi}\left(\alpha^{*}\right)$. Indeed, it follows from Lemma 2 that $\lim _{n \rightarrow \infty} r_{n}=r\left(\alpha^{*}\right)$, since the function $G(r)$, which was so defined that the number $r_{n}$ is the solution of the equation $G(r)=\alpha(n)$, is a continuous and strictly monotone function. Then it follows from Lemma 1 that the relation $\lim _{n \rightarrow \infty} \bar{\varphi}_{n}=\bar{\varphi}\left(\alpha^{*}\right)$ also holds.

We want to make a Taylor expansion of the function $\beta_{j}\left(r_{n}, \varphi\right)$ defined in (2.10) in the variable $\varphi$ around the point $\left(r_{n}, \bar{\varphi}_{n}\right)$. For this end, we introduce 
some notations. Put

$$
\begin{aligned}
& \eta_{j}^{(0)}=\eta_{j}^{(0)}(n)=\Re\left(\beta_{j}\left(r_{n}, \bar{\varphi}_{n}\right)-E \beta_{j}\left(r_{n}, \bar{\varphi}_{n}\right)\right) \\
& =\frac{1}{2} \log \left(r_{n}^{2}+\xi_{j}^{2}+2 r_{n} \xi_{j} \cos \bar{\varphi}_{n}\right) \\
& -\frac{1}{2} E \log \left(r_{n}^{2}+\xi^{2}+2 r_{n} \xi \cos \bar{\varphi}_{n}\right), \\
& \zeta_{j}^{(0)}=\zeta_{j}^{(0)}(n)=\Im\left(\beta_{j}\left(r_{n}, \bar{\varphi}_{n}\right)-E \beta_{j}\left(r_{n}, \bar{\varphi}_{n}\right)\right) \\
& =\arccos \frac{r_{n} \cos \bar{\varphi}_{n}+\xi_{j}}{\left(r_{n}^{2}+\xi_{j}^{2}+2 r_{n} \xi_{j} \cos \bar{\varphi}_{n}\right)^{1 / 2}} \\
& -E \arccos \frac{r_{n} \cos \bar{\varphi}_{n}+\xi}{\left(r_{n}^{2}+\xi^{2}+2 r_{n} \xi \cos \bar{\varphi}_{n}\right)^{1 / 2}}, \\
& \eta_{j}^{(1)}=\eta_{j}^{(1)}(n)=\left.\frac{\partial}{\partial \varphi} \Re\left(\beta_{j}\left(r_{n}, \varphi\right)-E \beta_{j}\left(r_{n}, \varphi\right)\right)\right|_{\varphi=\bar{\varphi}_{n}} \\
& =\left.\frac{\partial}{\partial \varphi} \Re \beta_{j}\left(r_{n}, \varphi\right)\right|_{\varphi=\bar{\varphi}_{n}} \\
& =-\frac{r_{n} \xi_{j} \sin \bar{\varphi}_{n}}{r_{n}^{2}+\xi_{j}^{2}+2 r_{n} \xi \cos \bar{\varphi}_{n}}, \\
& \zeta_{j}^{(1)}=\zeta_{j}^{(1)}(n)=\frac{\partial}{\partial \varphi} \Im\left(\beta_{j}\left(r_{n}, \bar{\varphi}_{n}\right)-E \beta_{j}\left(r_{n}, \bar{\varphi}_{n}\right)\right) \\
& =\left.\frac{\partial}{\partial \varphi} \Im \beta_{j}\left(r_{n}, \varphi\right)\right|_{\varphi=\bar{\varphi}_{n}} \\
& =\frac{r_{n} \xi_{j} \cos \bar{\varphi}_{n}+r_{n}^{2}}{r_{n}^{2}+\xi_{j}^{2}+2 r_{n} \xi_{j} \cos \bar{\varphi}_{n}}-\alpha(n) .
\end{aligned}
$$

[In the last identity we applied the same calculation as in (2.11).]

$$
\begin{aligned}
A_{0} & =A_{0}(n)=E \Re \beta_{j}\left(r_{n}, \bar{\varphi}_{n}\right) \\
& =\frac{1}{2} E \log \left(r_{n}^{2}+\xi^{2}+2 r_{n} \xi \cos \bar{\varphi}_{n}\right)-\alpha(n) \log r_{n}, \\
B_{0} & =B_{0}(n)-E \Im \beta_{j}\left(r_{n}, \bar{\varphi}_{n}\right) \\
& =E \arccos \frac{r_{n} \cos \bar{\varphi}_{n}+\xi}{\left(r_{n}^{2}+\xi^{2}+2 r_{n} \xi \cos \bar{\varphi}_{n}\right)^{1 / 2}}-\alpha(n) \bar{\varphi}_{n},
\end{aligned}
$$


the numbers $A_{2}=A_{2}(n)$ and $B_{2}=B_{2}(n)$ which are the second derivatives of the functions $E \Re \beta_{j}\left(r_{n}, \varphi\right)$ and $E \Im \beta_{j}\left(r_{n}, \varphi\right)$ in the point $\varphi=\bar{\varphi}_{n}$, that is,

$$
\begin{aligned}
& A_{2}=A_{2}(n)=-E \frac{r_{n} \xi \cos \bar{\varphi}_{n}}{U\left(r_{n}, \xi, \bar{\varphi}_{n}\right)}-2 E \frac{r_{n}^{2} \xi^{2} \sin ^{2} \bar{\varphi}_{n}}{U\left(r_{n}, \xi, \bar{\varphi}_{n}\right)^{2}}, \\
& B_{2}=B_{2}(n)=-E \frac{r_{n} \xi \sin \bar{\varphi}_{n}}{U\left(r_{n}, \xi, \bar{\varphi}_{n}\right)}+2 E \frac{r_{n} \xi \sin \bar{\varphi}_{n}\left(r_{n} \xi \cos \bar{\varphi}_{n}+r^{2}\right)}{U\left(r_{n}, \xi, \bar{\varphi}_{n}\right)^{2}},
\end{aligned}
$$

$$
\begin{aligned}
\eta_{j}^{(2)} & =\eta_{j}^{(2)}(n)=\left.\Re \frac{\partial^{2}}{\partial \varphi^{2}}\left(\beta_{j}\left(r_{n}, \varphi\right)-E \beta_{j}\left(r_{n}, \varphi\right)\right)\right|_{\varphi=\bar{\varphi}_{n}} \\
& =-\frac{r_{n} \xi_{j} \cos \bar{\varphi}_{n}}{U\left(r_{n}, \xi_{j}, \bar{\varphi}_{n}\right)}-2 \frac{r_{n}^{2} \xi_{j}^{2} \sin ^{2} \bar{\varphi}_{n}}{U\left(r_{n}, \xi_{j}, \bar{\varphi}_{n}\right)^{2}}-A_{2}
\end{aligned}
$$

$$
\begin{aligned}
\zeta_{j}^{(2)} & =\zeta_{j}^{(2)}(n)=\left.\Im \frac{\partial^{2}}{\partial \varphi^{2}}\left(\beta_{j}\left(r_{n}, \varphi\right)-E \beta_{j}\left(r_{n}, \varphi\right)\right)\right|_{\varphi=\bar{\varphi}_{n}} \\
& =\frac{-r_{n} \xi_{j} \sin \bar{\varphi}_{n}}{U\left(r_{n}, \xi_{j}, \bar{\varphi}_{n}\right)}+2 \frac{r_{n} \xi_{j} \sin \bar{\varphi}_{n}\left(r_{n} \xi_{j} \cos \bar{\varphi}_{n}+r_{n}^{2}\right)}{U\left(r_{n}, \xi_{j}, \bar{\varphi}_{n}\right)^{2}}-B_{2} .
\end{aligned}
$$

We can write

$$
\begin{aligned}
\Re \beta_{j}\left(r_{n}, \varphi\right)= & A_{0}+\eta_{j}^{(0)}\left(r_{n}, \bar{\varphi}_{n}\right)+\eta_{j}^{(1)}\left(r_{n}, \bar{\varphi}_{n}\right)\left(\varphi-\bar{\varphi}_{n}\right) \\
& +\frac{1}{2}\left(A_{2}+\eta_{j}^{(2)}\right)\left(\varphi-\bar{\varphi}_{n}\right)^{2}+\frac{1}{6} \vartheta_{j, 1}\left(\varphi-\bar{\varphi}_{n}\right)^{3}, \\
\Im \beta_{j}\left(r_{n}, \varphi\right)= & B_{0}+\zeta_{j}^{(0)}\left(r_{n}, \bar{\varphi}_{n}\right)+\zeta_{j}^{(1)}\left(r_{n}, \bar{\varphi}_{n}\right)\left(\varphi-\bar{\varphi}_{n}\right) \\
& +\frac{1}{2}\left(B_{2}+\zeta_{j}^{(2)}\right)\left(\varphi-\bar{\varphi}_{n}\right)^{2}+\frac{1}{6} \vartheta_{j, 2}\left(\varphi-\bar{\varphi}_{n}\right)^{3},
\end{aligned}
$$

where

$$
\begin{aligned}
& \vartheta_{j, 1}=\vartheta_{j, 1}\left(r_{n}, \varphi\right)=\left.\frac{\partial^{3}}{\partial \varphi^{3}} \Re \beta_{j}\left(r_{n}, \varphi\right)\right|_{\varphi=\tilde{\varphi}}, \\
& \vartheta_{j, 2}=\vartheta_{j, 2}\left(r_{n}, \varphi\right)=\left.\frac{\partial^{3}}{\partial \varphi^{3}} \Im \beta_{j}(r, \varphi)\right|_{\varphi=\tilde{\varphi}}
\end{aligned}
$$

with some numbers $\tilde{\varphi}$ and $\tilde{\tilde{\varphi}}$ in the interval $\left[\varphi, \bar{\varphi}_{n}\right]$. Summing up the last relations for $j=1, \ldots, n$, we get the following relation for the function $Z_{n}\left(r_{n}, \varphi\right)$ 
defined in (2.9):

$$
\begin{aligned}
Z_{n}(r, \varphi)= & n\left(A_{0}+i B_{0}\right)+\sqrt{n} S_{0}(n)+i T_{0}(n) \\
& +\sqrt{n}\left(S_{1}(n)+i T_{1}(n)\right)\left(\varphi-\bar{\varphi}_{n}\right) \\
& +\frac{n}{2}\left(A_{2}+i B_{2}\right)\left(\varphi-\bar{\varphi}_{n}\right)^{2} \\
& +\frac{\sqrt{n}}{2}\left(\varepsilon_{1}(n)+i \varepsilon_{2}(n)\right)\left(\varphi-\bar{\varphi}_{n}\right)^{2} \\
& +\frac{n}{6}\left(\delta_{1}(n)+i \delta_{2}(n)\right)\left(\varphi-\bar{\varphi}_{n}\right)^{3}
\end{aligned}
$$

where

$$
\begin{gathered}
S_{0}=S_{0}(n)=\frac{1}{\sqrt{n}} \sum_{j=1}^{n} \eta_{j}^{(0)}\left(r_{n}, \bar{\varphi}_{n}\right) \text { and } \\
T_{0}=T_{0}(n)=\sum_{j=1}^{n} \zeta_{j}^{(0)}\left(r_{n}, \bar{\varphi}_{n}\right) \bmod 2 \pi, \\
S_{1}=S_{1}(n)=\frac{1}{\sqrt{n}} \sum_{j=1}^{n} \eta_{j}^{(1)}\left(r_{n}, \bar{\varphi}_{n}\right), \\
T_{1}=T_{1}(n)=\frac{1}{\sqrt{n}} \sum_{j=1}^{n} \zeta_{j}^{(1)}\left(r_{n}, \bar{\varphi}_{n}\right),
\end{gathered}
$$

and

$$
\begin{aligned}
& \varepsilon_{1}(n)=\frac{1}{\sqrt{n}} \sum_{j=1}^{n} \eta_{j}^{(2)}\left(r_{n}, \bar{\varphi}_{n}\right), \quad \varepsilon_{2}(n)=\frac{1}{\sqrt{n}} \sum_{j=1}^{n} \zeta_{j}^{(2)}\left(r_{n}, \bar{\varphi}_{n}\right), \\
& \delta_{k}(n)=\frac{1}{n} \sum_{j=1}^{n} \vartheta_{j, k}\left(r_{n}, \varphi\right), \quad k=1,2 .
\end{aligned}
$$

We want to give a good asymptotic formula for the integral (2.8) by means of (4.7) if Property B holds. Define the intervals

$$
\bar{I}(n)=\left[\bar{\varphi}_{n}-n^{-1 / 2+1 / 10}, \bar{\varphi}_{n}+n^{-1 / 2+1 / 10}\right] \quad \text { and } \quad I(n)=\bar{I}(n) \cap[0, \pi) .
$$

Observe that for sufficiently large $n \bar{I}(n)=I(n)$ if $0<\bar{\varphi}\left(\alpha^{*}\right)<\pi$, and $\bar{I}(n)=$ $I(n) \cup(-I(n))$ if $\bar{\varphi}\left(\alpha^{*}\right)=0$ or $\bar{\varphi}\left(\alpha^{*}\right)=\pi$ with $\alpha^{*}=\lim _{n \rightarrow \infty} \alpha(n)$. This relation follows from Lemma 1, the relation $\lim _{n \rightarrow \infty} \bar{\varphi}_{n}=\bar{\varphi}\left(\alpha^{*}\right)$ which we pointed out at the beginning of this Section, Property B and the observation that in the case $\bar{\varphi}\left(\alpha^{*}\right)=0$ or $\pi K^{ \pm}\left(r\left(\alpha^{*}\right)\right)>0$ with a strict inequality. Indeed, the inequality $K^{ \pm}(r(\alpha(n)))>0$ also holds in this case. These facts imply the relation between the intervals $I(n)$ and $\bar{I}(n)$ formulated in this paragraph.

We claim that there is an appropriate set $\Omega(n)$ on the probability space where the random variables $\xi_{1}, \xi_{2}, \ldots$ are defined such that

$$
P(\Omega(n)) \rightarrow 1 \text { as } n \rightarrow \infty,
$$


and

$$
\Re\left(\frac{1}{\pi} \int_{I(n)} \exp \left\{Z_{n}\left(r_{n}, \varphi\right)\right\} d \varphi\right)= \begin{cases}2 D_{n}, & \text { if } 0<\bar{\varphi}_{n}<\pi, \\ D_{n}, & \text { if } \bar{\varphi}_{n}=0 \text { or } \bar{\varphi}=\pi\end{cases}
$$

on the set $\Omega(n)$ for the function $Z_{n}(r, \varphi)$ defined in (2.9) with a (random) number $D_{n}$ which satisfies the relation

$$
D_{n}=\Re\left(\frac{\sqrt{\pi} \exp \left\{Z_{n}\left(r_{n}, \bar{\varphi}_{n}\right)-\frac{\left(S_{1}(n)+i T_{1}(n)\right)^{2}}{2\left(A_{2}+i B_{2}\right)}+O\left(n^{-1 / 10}\right)\right\}}{\sqrt{2 n\left(-A_{2}-i B_{2}\right)}}\right),
$$

where $S_{1}(n)$ and $T_{1}(n)$ are defined in (4.9), $A_{0}, B_{0}$ in (4.3), $\bar{\varphi}_{n}=\bar{\varphi}(\alpha(n))$ and $\sqrt{\left(-A_{2}-i B_{2}\right)}$ is meant as the square root with positive real part. Let us remark that $A_{2}<0$, which statement is proved with a slightly different notation in Lemma 3. Moreover, the numbers $A_{2}(n)$ are strictly separated from zero for all sufficiently large $n$ since $\left(r_{n}, \bar{\varphi}_{n}\right) \rightarrow\left(r\left(\alpha^{*}\right), \bar{\varphi}\left(\alpha^{*}\right)\right)$ as $n \rightarrow \infty$, and Property A and Lemma A can be applied if $\bar{\varphi}\left(\alpha^{*}\right)=0$ or $\bar{\varphi}\left(\alpha^{*}\right)=\pi$. We also claim that

the angle between the complex numbers

$$
\frac{\exp \left\{Z_{n}\left(r_{n}, \bar{\varphi}_{n}\right)-\left(\left(S_{1}(n)+i T_{1}(n)\right)^{2} / 2\left(A_{2}+i B_{2}\right)\right)\right\}}{\sqrt{2 n\left(-A_{2}-i B_{2}\right)}}
$$

and $i=\sqrt{-1}$ is larger than $n^{-1 / 20}$

and

$$
\left|\int_{[0, \pi] \backslash(I(n))} \exp \left\{Z_{n}\left(r_{n}, \varphi\right)\right\} d \varphi\right|=O\left(\exp \left\{\Re Z_{n}\left(r_{n}, \bar{\varphi}_{n}\right)-\text { const. } n^{1 / 5}\right\}\right),
$$

and the $O(\cdot)$ is uniform in (4.11) and (4.12) on the sets $\Omega(n)$.

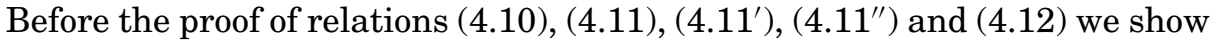
that they imply Lemma 4 . First we show by a comparison of the right-hand side of $(4.11),\left(4.11^{\prime}\right),\left(4.11^{\prime \prime}\right)$ and (4.12) that a negligible error is committed on the set $\Omega(n)$ if the integral (2.8) is restricted to the set $I(n)$; that is, the expression $D_{n}$ or $2 D_{n}$ defined in $\left(4.11^{\prime}\right)$ is a good approximation of $S^{(k)}(n)$.

Formula (4.15) which will appear in the definition of the set $\Omega(n)$ implies that

$$
\left|\frac{\left(S_{1}(n)+i T_{1}(n)\right)^{2}}{2\left(A_{2}+i B_{2}\right)}\right|<\text { const. } n^{1 / 10}
$$

on the set $\Omega(n)$. This relation together with $\left(4.11^{\prime}\right),\left(4.11^{\prime \prime}\right)$ imply that

$$
\begin{aligned}
\left|D_{n}\right| & \geq \text { const. }\left|\Re\left(\frac{\exp \left\{Z_{n}\left(r_{n}, \bar{\varphi}_{n}\right)-\left(\left(S_{1}(n)+i T_{1}(n)\right)^{2} / 2\left(A_{2}+i B_{2}\right)\right)\right\}}{\sqrt{2 n\left(-A_{2}-i B_{2}\right)}}\right)\right| \\
& \geq n^{-1 / 20} \text { const. }\left|\frac{\exp \left\{Z_{n}\left(r_{n}, \bar{\varphi}_{n}\right)-\left(\left(S_{1}(n)+i T_{1}(n)\right)^{2} / 2\left(A_{2}+i B_{2}\right)\right)\right\}}{\sqrt{2 n\left(-A_{2}-i B_{2}\right)}}\right| \\
& \geq \exp \left\{\Re Z_{n}\left(r_{n}, \bar{\varphi}_{n}\right)-\text { const. } n^{1 / 10}\right\} .
\end{aligned}
$$


The above estimate together with (4.11), (4.12) and the definition of $S^{(k)}(n)$ imply that $S^{(k)}(n)=2 D_{n}(1+o(1))$ if $0<\bar{\varphi}\left(\alpha^{*}\right)<\pi$ and $S^{(k)}(n)=D_{n}(1+o(1))$ if $\bar{\varphi}\left(\alpha^{*}\right)=0$ or $\bar{\varphi}\left(\alpha^{*}\right)=\pi$. Hence to prove Lemma 4 it is enough to give a good estimate on $D_{n}$. We shall consider the cases $0<\bar{\varphi}\left(\alpha^{*}\right)<\pi, \bar{\varphi}\left(\alpha^{*}\right)=0$ and $\bar{\varphi}\left(\alpha^{*}\right)=\pi$ separately. We get with the help of relation $\left(4.11^{\prime}\right)$ and the identity $Z_{n}\left(r_{n}, \bar{\varphi}_{n}\right)=n\left(A_{0}+i B_{0}\right)+\sqrt{n} S_{0}(n)+i T_{0}(n)$ that on the set $\Omega(n)$,

$$
\begin{aligned}
D_{n}= & \frac{\sqrt{2}}{\sqrt{K n \pi}} \exp \left\{n A_{0}+\sqrt{n} S_{0}-U_{1}\right\} \cos \left(n B_{0}+T_{0}-U_{2}-\frac{\omega}{2}\right) \\
& \times\left(1+O\left(n^{-1 / 10}\right)\right) \text { if } 0<\bar{\varphi}\left(\alpha^{*}\right)<\pi
\end{aligned}
$$

with

$$
\begin{aligned}
& U_{1}=U_{1}(n)=\frac{A_{2}\left(S_{1}^{2}-T_{1}^{2}\right)+2 B_{2} S_{1} T_{1}}{2\left(A_{2}^{2}+B_{2}^{2}\right)} \\
& U_{2}=U_{2}(n)=\frac{-B_{2}\left(S_{1}^{2}-T_{1}^{2}\right)+2 A_{2} S_{1} T_{1}}{2\left(A_{2}^{2}+B_{2}^{2}\right)}
\end{aligned}
$$

and

$$
K=K(n)=\left(A_{2}^{2}+B_{2}^{2}\right)^{1 / 2}, \quad \omega=\omega(n)=\arctan \frac{B_{2}}{A_{2}}
$$

because of the relation

$$
\frac{\left(S_{1}+i T_{1}\right)^{2}}{2\left(A_{2}+i B_{2}\right)}=U_{1}+i U_{2}
$$

In the case $\bar{\varphi}\left(\alpha^{*}\right)=0, B_{0}=0, B_{2}=0, T_{0}=0$ and $S_{1}=0$, hence

$$
D_{n}=\frac{1}{\sqrt{2 \pi\left|A_{2}\right| n}} \exp \left\{\frac{T_{1}^{2}}{2 A_{2}}+n A_{0}+\sqrt{n} S_{0}\right\}\left(1+O\left(n^{-1 / 10}\right)\right)
$$

$$
\text { if } \bar{\varphi}\left(\alpha^{*}\right)=0
$$

and in the case $\bar{\varphi}\left(\alpha^{*}\right)=\pi, n B_{0}=n(-\pi-\alpha(n))=k(n) \pi, T_{0}=0$ and $S_{1}=0$. Hence

$$
\begin{array}{r}
D_{n}=(-1)^{k(n)} \frac{1}{\sqrt{2 \pi\left|A_{2}\right| n}} \exp \left\{\frac{T_{1}^{2}}{2 A_{2}}+n A_{0}+\sqrt{n} S_{0}\right\}\left(1+O\left(n^{-1 / 10}\right)\right) \\
\text { if } \bar{\varphi}\left(\alpha^{*}\right)=\pi .
\end{array}
$$

Lemma 4 follows from (4.13), $\left(4.13^{\prime}\right),\left(4.13^{\prime \prime}\right)$ and the relation between $S^{(k)}(n)$ and $D_{n}$. 
We define $\Omega(n)$ in the form $\Omega(n)=\Omega_{1}(n) \cap \Omega_{2}(n) . \Omega_{1}(n)$ is the set where the above relations hold:

$$
\begin{aligned}
& \left|S_{1}(n)\right|<n^{1 / 20}, \\
& \left|T_{1}(n)\right|<n^{1 / 20}, \\
& \left|\varepsilon_{k}(n)\right|<n^{1 / 10}, \quad k=1,2, \\
& \left|\bar{\delta}_{k}(n)\right|<n^{1 / 10}, \quad k=1,2, \\
& \mid \sum_{j=1}^{n} \frac{\xi_{j} \cos \left(\bar{\varphi}_{n} \pm n^{-4 / 10}\right)}{r_{n}^{2}+\xi_{j}^{2}+2 r_{n} \xi_{j} \cos \left(\bar{\varphi}_{n} \pm n^{-4 / 10}\right)} \\
& \quad-n E \frac{\xi \cos \left(\bar{\varphi}_{n} \pm n^{-4 / 10}\right)}{r_{n}^{2}+\xi^{2}+2 r_{n} \xi \cos \left(\bar{\varphi}_{n} \pm n^{-4 / 10}\right)} \mid<n^{11 / 20},
\end{aligned}
$$

where

$$
\bar{\delta}_{k}(n)=\frac{1}{n} \sum_{j=1}^{n} \bar{\vartheta}_{j, k}, \quad k=1,2
$$

with

$$
\begin{aligned}
\bar{\vartheta}_{j, 1} & =\sup _{\left|\varphi-\bar{\varphi}_{n}\right|<n^{-1 / 2+1 / 10}}\left|\frac{1}{2} \frac{\partial^{3}}{\partial \varphi^{3}}\left(\log \left(r_{n}^{2}+\xi_{j}^{2}+2 r_{n} \xi_{j} \cos \varphi\right)\right)\right|, \\
\bar{\vartheta}_{j, 2} & =\sup _{\left|\varphi-\bar{\varphi}_{n}\right|<n^{-1 / 2+1 / 10}}\left|\frac{\partial^{3}}{\partial \varphi^{3}} \arccos \frac{r_{n} \cos \varphi+\xi}{\left(r_{n}^{2}+\xi^{2}+2 r_{n} \xi \cos \varphi\right)^{1 / 2}}\right| .
\end{aligned}
$$

The set $\Omega_{2}(n)$ is defined as the set where the above relation holds:

$$
\begin{aligned}
& \left|W(n)-\frac{1}{2}\right|>n^{-1 / 20 \quad \text { with }} \\
& \begin{array}{l}
W(n)=\frac{1}{\pi}\left(n B_{0}(n)+T_{0}(n)-U_{2}(n)-\frac{\omega(n)}{2}\right) \quad \bmod 1 \\
\text { if } 0<\bar{\varphi}\left(\alpha^{*}\right)<\pi,
\end{array}
\end{aligned}
$$

where $B_{0}, T_{0}, U_{2}$ and $\omega$ are defined in (4.1), (4.2), (4.14) and (4.14').

The above defined set $\Omega(n)$ satisfies relation (4.10), since both $\Omega_{1}(n)$ and $\Omega_{2}(n)$ satisfy it. It holds for $\Omega_{1}(n)$ since the random variables $\sqrt{n} S_{1}(n)$, $\sqrt{n} T_{1}(n), \sqrt{n} \varepsilon_{k}(n), k=1,2$, and the last expression in (4.15) are sums of $n$ independent random variables with expectation zero and finite second moment, while $n \bar{\delta}_{k}(n)$ is the sum of $n$ independent random variables with finite expectation. Hence we can deduce relation (4.15) from the Chebyshev and Markov inequalities if we know that the appropriate variances and expected value have a uniform bound for all sufficiently large $n$. But this holds because of relation (2.5) and the fact that $z\left(\alpha^{*}\right)=r\left(\alpha^{*}\right) \exp \left(i \bar{\varphi}\left(\alpha^{*}\right)\right)$ and $z_{n}=r_{n} \exp \left(i \bar{\varphi}_{n}\right)$ are separated from the real line if $0<\bar{\varphi}\left(\alpha^{*}\right)<\pi$, they are separated from $-\Sigma$ if $\bar{\varphi}\left(\alpha^{*}\right)=0$, and from $\Sigma$ if $\bar{\varphi}\left(\alpha^{*}\right)=\pi$. The last observation 
is needed to check that the singularity of the random functions in the point $r_{n}$ or $-r_{n}$ makes no problem.

The probability of the event that relation $\left(4.15^{\prime}\right)$ holds tends to 1 , as $n \rightarrow \infty$. This follows from Proposition B which will be proved in Section 5. Indeed, it follows from Proposition B that the random variables $W(n)$ converge in distribution to the uniform distribution if $n \rightarrow \infty$, and this implies $\left(4.15^{\prime}\right)$. The above mentioned limit theorem holds because the vectors $\left(T_{0}(n), S_{1}(n), T_{1}(n)\right)$ converge in distribution to a random vector $\left(T_{0}, S_{1}, S_{2}\right)$ such that $T_{0}$ is uniformly distributed mod 1 , and the vector $\left(S_{1}, S_{2}\right)$ is independent of $T_{0}$. The limit distribution for $W(n)$ follows from this fact and the definition of $W(n)$.

Formula $\left(4.11^{\prime \prime}\right)$ follows from $\left(4.14^{\prime \prime}\right)$ and $\left(4.15^{\prime}\right)$. To prove relation (4.11) and $\left(4.11^{\prime}\right)$ in the case $0<\bar{\varphi}\left(\alpha^{*}\right)<\pi$, observe that by (4.7) and the definition of the set $\Omega(n)$,

$$
\begin{aligned}
Z_{n}\left(r_{n}, \varphi\right)-Z_{n}\left(r_{n}, \bar{\varphi}_{n}\right)= & n\left(A_{2}+i B_{2}\right) \frac{\left(\varphi-\bar{\varphi}_{n}\right)^{2}}{2} \\
& +\sqrt{n}\left(S_{1}(n)+i T_{1}(n)\right)\left(\varphi-\bar{\varphi}_{n}\right) \\
& +O\left(n^{-1 / 10}\right) \\
= & \frac{n\left(A_{2}+i B_{2}\right)}{2}\left(\varphi-\bar{\varphi}_{n}+\frac{S_{1}+i T_{1}}{\sqrt{n}\left(A_{2}+i B_{2}\right)}\right)^{2} \\
& -\frac{\left(S_{1}+i T_{1}\right)^{2}}{2\left(A_{2}+i B_{2}\right)}+O\left(n^{-1 / 10}\right)
\end{aligned}
$$

if $\varphi \in I(n)$ and $\omega \in \Omega(n)$, hence

$$
\begin{aligned}
& \int_{I(n)} \exp \left\{Z_{n}\left(r_{n}, \varphi\right)-Z_{n}\left(r_{n}, \bar{\varphi}_{n}\right)\right\} d \varphi \\
&= \int_{I(n)} \exp \left\{\frac{n\left(A_{2}+i B_{2}\right)}{2}\left(\varphi-\bar{\varphi}_{n}+\frac{S_{1}+i T_{1}}{\sqrt{n}\left(A_{2}+i B_{2}\right)}\right)^{2}\right. \\
&\left.-\frac{\left(S_{1}+i T_{1}\right)^{2}}{2\left(A_{2}+i B_{2}\right)}+O\left(n^{-1 / 10}\right)\right\} d \varphi \\
&= \int_{-\infty}^{\infty} \exp \left\{\frac{n\left(A_{2}+i B_{2}\right)}{2}\left(\varphi-\bar{\varphi}_{n}+\frac{S_{1}+i T_{1}}{\sqrt{n}\left(A_{2}+i B_{2}\right)}\right)^{2}\right. \\
&\left.-\frac{\left(S_{1}+i T_{1}\right)^{2}}{2\left(A_{2}+i B_{2}\right)}+O\left(n^{-1 / 10}\right)\right\} d \varphi+O\left(\exp \left(-K n^{1 / 5}\right)\right) \\
&= \sqrt{2 \pi} \exp \left\{-\left(\left(S_{1}+i T_{1}\right)^{2} / 2\left(A_{2}+i B_{2}\right)\right)+O\left(n^{-1 / 10}\right)\right\} \\
& \sqrt{\left(-A_{2}-i B_{2}\right) n}
\end{aligned}
$$

since $\int_{\infty}^{\infty} \exp \left(-A(\varphi-B)^{2}\right) d \varphi=\sqrt{\pi / A}$ if $\Re A>0$, and the main term in the middle expression of the last relation is dominating, being larger than $O\left(\exp \left(-\right.\right.$ const $\left.\left.n^{1 / 10}\right)\right)$. In the above calculation we have exploited that $A_{2}<0$. 
The expression $\sqrt{\left(-A_{2}-i B_{2}\right)}$ is meant as the square root with positive real part.

The cases $\bar{\varphi}\left(\alpha^{*}\right)=0$ or $\bar{\varphi}\left(\alpha^{*}\right)=\pi$ are similar, but simpler. The integrals we are interested in can be calculated similarly, only the approximating integrals $\int_{-\infty}^{\infty}$ must be replaced by $\int_{0}^{\infty}$ or $\int_{-\infty}^{0}$. (We exploit during these calculations that $S_{1}=0$ in the present case.) The main part of the integral under consideration is real, since $S_{1}=0, B_{2}=0, T_{0}=0$ and $B_{0}=0 \bmod \pi$ in this case.

To prove (4.12) it is enough to show that

$$
\Re Z_{n}\left(r_{n}, \varphi\right) \leq \Re Z_{n}\left(r_{n}, \bar{\varphi}_{n}\right)-\text { const. } n^{1 / 5} \quad \text { if } \varphi \in[0, \pi] \backslash I(n)
$$

on the set $\Omega(n)$, where the function $Z_{n}\left(r_{n}, \varphi\right)$ is defined in (2.9) and (2.10). First we show the following weaker result:

$$
\Re Z_{n}\left(r_{n}, \bar{\varphi}_{n} \pm n^{-2 / 5}\right)<\Re Z_{n}\left(r_{n}, \bar{\varphi}_{n}\right)-\text { const. } n^{1 / 5},
$$

that is, relation (4.17) holds if some very special points of the set $[0, \pi] \backslash I(n)$ are considered.

To prove relation (4.18) let us first observe that for $A_{2}=A_{2}(n)$ defined in (4.4), $A_{2}<-K$ with some negative constant $K$. Indeed, either $0<\bar{\varphi}<\pi$ in which case $A_{2}=-2 E\left(r_{n}^{2} \xi^{2} \sin ^{2} \bar{\varphi}_{n} / U\left(r_{n}, \xi, \bar{\varphi}_{n}\right)^{2}\right)<-K$ because of Lemma 1 or $\bar{\varphi}_{n}=0$ or $\bar{\varphi}_{n}=\pi$, and in these cases $A_{2}=E\left(\mp r_{n} \xi /\left(r_{n} \pm \xi\right)^{2}\right)<-K$ because of Property B. We get relation (4.18) by taking the real part of the first identity in (4.16) with the choice $\varphi=\bar{\varphi}_{n} \pm n^{-2 / 5}$ with the help of the following observations: $n A_{2}\left(\left(\varphi-\bar{\varphi}_{n}\right)^{2} / 2\right)<-$ const. $n^{1 / 5}, \sqrt{n}\left|\left(\varphi-\bar{\varphi}_{n}\right) S_{1}(n)\right|<n^{3 / 20}$ on the set $\Omega(n)$ because of the relation $A_{2}<-K$ and (4.15).

Relation (4.17) can be rewritten, with the change of variable $\psi=\cos \varphi$, in the equivalent form,

$$
Y_{n}(\psi) \leq Y_{n}\left(\cos \bar{\varphi}_{n}\right)-\text { const. } n^{1 / 5} \quad \text { if }\left|\arccos \psi-\bar{\varphi}_{n}\right| \geq n^{-2 / 5},
$$

on the set $\Omega(n)$, with the function $Y_{n}(\psi)$ defined as

$$
Y_{n}(\psi)=\Re Z_{n}\left(r_{n}, \arccos \psi\right)=\sum_{j=1}^{n} \frac{1}{2} \log \left(r_{n}^{2}+\xi_{j}^{2}+2 r_{n} \xi_{j} \psi\right)-n \alpha(n) \log r_{n} .
$$

Relation (4.18) implies that

$$
Y_{n}\left(\cos \left(\bar{\varphi}_{n} \pm n^{-2 / 5}\right)\right) \leq Y_{n}\left(\cos \bar{\varphi}_{n}\right)-\text { const. } n^{1 / 5} .
$$

To prove (4.19) it is enough to observe that

$$
\frac{d^{2}}{d \psi^{2}} Y_{n}(\psi)=-\sum_{j=1}^{n} \frac{2 r_{n}^{2} \xi_{j}^{2}}{\left(r_{n}^{2}+\xi_{j}^{2}+2 r_{n} \xi_{j} \psi\right)^{2}} \leq 0,
$$

hence the function $Y_{n}(\cdot)$ is concave, and relation (4.20) implies its strengthened form, relation (4.19). 


\section{Proof of the limit theorems for sums of independent vectors.}

Proof of Proposition A. In the proof we apply a natural adaptation of the characteristic function technique. We shall investigate the expressions

$$
\varphi(t, l)=E \exp \left\{i t X_{1}+2 \pi i l\left(Y_{1}-\alpha\right)\right\},
$$

where $t \in R^{k}, l$ is an arbitrary integer if $G_{0}=G, l$ is an integer, $0 \leq l<p$ if $G_{0}=\{j / p, j=0, \ldots, p-1\}$, and $t X_{s}$ denotes scalar product. We claim that

$$
\begin{aligned}
\varphi(t, l) & =\exp \left\{-\frac{1}{2} t \Sigma t^{*}+o\left(t^{2}\right)\right\} \quad \text { if } l=0 \text { and } t \rightarrow 0, \\
|\varphi(t, l)| & <C<1 \quad \text { if } l \neq 0 \text { and }|t|<\varepsilon,
\end{aligned}
$$

where the constants $C<1$ and $\varepsilon>0$ may depend on $l$,

Since $E X_{1}=0$, and the coefficient of $Y_{1}-\alpha$ in the definition of the function $\varphi(t, l)$ is zero for $l=0$, the first line of relation (5.2) follows from a simple Taylor expansion, just as it is done in the proof of the classical central limit theorem. First we prove the second line of (5.2) first in the case if $G_{0}=G$, that is, if the minimal coset containing the support $\mu$ is the whole group $G$. We show that in this case for all positive integers $l$ and $0 \leq \alpha \leq 1$ there is some $\delta=\delta(l)>0$ and $\eta=\eta(l)>0$ depending only on $l$ such that the distribution $\mu$ of $Y_{s}$ satisfies the inequality

$$
\mu\left(\bigcup_{j=1}^{l}\left[\frac{j}{l}-\eta+\alpha, \frac{j}{l}+\eta+\alpha\right]\right)<1-\delta .
$$

Let us emphasize that the numbers $\eta>0$ and $\delta>0$ in (5.3) may depend on $l$ but not on $\alpha$.

To prove (5.3) first we show that for all sets,

$$
A(\beta)=A(\beta, l, \eta)=\bigcup_{j=1}^{l}\left(\frac{j}{l}+\beta-2 \eta, \frac{j}{l}+\beta+2 \eta\right),
$$

$\mu(A(\beta))<1-\delta$ if the numbers $\eta=\eta(\beta, l)$ and $\delta=\delta(\beta, l)$ are appropriately chosen. Indeed, the $\mu$ measure of the (finite) sets $\bigcup_{j=1}^{l}\{j / l+\beta\}$ is less than one for all $0 \leq \beta \leq 1$, since otherwise the support of the measure $\mu$ were concentrated on a finite coset. Since these sets are compact, this relation also holds for their sufficiently small neighborhoods.

Since the group $G$ is compact, there is a finite cover of $G$ with some sets of the form $\bar{A}(\beta)$, which sets are defined in the same way as $A(\beta),(\mu(A(\beta)<$ $1-\delta(\beta)$ ), only $2 \eta$ is replaced by $\eta$ in their definition. If we choose $\eta$ as the minimum of the numbers $\eta$ appearing in the definition of the sets $\bar{A}(\beta)$ appearing in this finite cover, then all sets which are considered at the left-hand side of (5.3) are contained in one of the sets $A(\beta)$ for which $\bar{A}(\beta)$ takes part in this cover. Hence relation (5.3) holds if $\eta$ and $\delta$ are chosen as the minimum of those values $\eta(\beta)>0$ and $\delta(\beta)>0$ which appear in the sets $A(\beta)$ for which $\bar{A}(\beta)$ takes part in this finite cover of $G$. 
Relation (5.3) implies that for sufficiently small $\varepsilon=\varepsilon(l)>0$,

$$
P\left(\left|l Y_{1}+t X_{1}-\alpha\right|>\frac{\eta}{2}\right)>\frac{\delta}{2},
$$

for all $0 \leq \alpha \leq 1$ if $t<\varepsilon$ with some $\eta=\eta(l)$ and $\delta=\delta(l)$. Hence

$$
E \Re \exp \left(i\left(l Y_{1}+t X_{1}-\alpha\right)\right)<1-\frac{\eta \delta^{2}}{8}
$$

for all $\alpha \in[0,1]$. Since this relation holds for all $\alpha$, this implies the second line of (5.2) in the case $G=G_{0}$.

If $G_{0}+\alpha$ with $G_{0}=\left\{\frac{j}{p}, j=0,1, \ldots, p-1\right\}$, is the minimal coset containing the support of $\mu$, then the distribution of $Y_{1}-\alpha$ is concentrated on $G_{0}$. The distribution of $Y_{1}-\alpha$ is concentrated on some points of the form $k_{u} / p, u=$ $0, \ldots, r$ with some $r$ such that the $\mu$ measure of all these points $k_{u} / p$ is positive. We may assume by replacing $\alpha$ by $\alpha-k_{0} / p$, if this is needed, that $k_{0}=$ 0 . Moreover, because of the minimality property of $G_{0}$ the greatest common divisor of $k_{1}, \ldots, k_{r}$ and $p$ equals 1 . Hence there are some integers $N_{u}, u=$ $1, \ldots, r$ and $N$ such that

$$
N p+\sum_{u=1}^{r} N_{u} k_{u}=1
$$

This fact implies that for any $1 \leq l<p$ all vectors $\exp \left\{2 \pi i l \frac{k_{u}}{p}\right\}, u=0, \ldots, r$ cannot be parallel. Indeed, otherwise the relation $l k_{u}=l k_{0}=0 \bmod p$ would hold for all $u=1, \ldots, r$, and this contradicts (5.4). Also, the maximum between the angles of the vectors $\exp \left\{i t X_{1}+2 \pi\left(i l k_{u} / p\right)\right\}$ are separated from zero with positive probability, and this fact implies the second line of (5.2) in this case, too.

Since $E \exp \left\{i t U_{n}+2 \pi i l V_{n}\right\}=\left[\varphi\left(t / \sqrt{n} X_{1}, l\right)\right]^{n}$, relation (5.2) implies that

$$
E \exp \left\{i t U_{n}+2 \pi i l V_{n}\right\}= \begin{cases}\exp \left\{-\frac{1}{2} t \Sigma t^{*}\right\}(1+o(1)), & \text { if } l=0 \\ o(1), & \text { if } l \neq 0 .\end{cases}
$$

Here $t \in R^{k}, l=0, \pm 1, \pm 2, \ldots$ if $G_{0}=G$, and $l$ is an integer, $0 \leq l<p$, if $G_{0}=$ $\{j / p, j=0, \ldots, p-1\}$. This means that $\lim _{n \rightarrow \infty} E \exp \left\{i t U_{n}+2 \pi i l V_{n}\right\}=$ $E \exp \{i t U+2 \pi i l V\}$ for all such $t$ and $l$, where $(U, V)$ is such a random vector whose distribution is described in Proposition A. This relation implies Proposition A.

Proof of Proposition B. The proof is a slight modification of that of Proposition A. It is enough to prove a modification of (5.2) under the condition of Proposition B where the characteristic function $\varphi(t, l)$ is replaced by $\varphi_{n}(t, l)=E \exp \left\{i t X_{1}(n)+2 \pi i l\left(Y_{1}(n)-\alpha\right)\right\}$. The constant $C<1$ in the second line of this modified relation (5.2) must not depend on $n$. The first line of this modified formula (5.2) holds, since it holds if $X_{1}(n)$ is replaced by $X$, and $\left(E \exp \left(\right.\right.$ it $\left.\left.X_{1}(n)\right)-E \exp (i t X)\right)=o\left(t^{2}\right)$ as $t \rightarrow 0$. The second line of 
(5.2) can be deduced from a modified version of (5.3) where the distribution $\mu$ of $Y_{1}$ is replaced by the distribution $\mu_{n}$ of $Y_{1}(n)$, but the numbers $\eta$ and $\delta$ must not depend on $n$. This can be deduced, just as it was done in the proof of Proposition A, from the weaker relation $\mu_{n}(A(\beta))<1-\delta$ with

$$
A(\beta)=A(\beta, l, \eta)=\bigcup_{j=1}^{l}\left(\frac{j}{l}+\beta-2 \eta, \frac{j}{l}+\beta+2 \eta\right),
$$

$\delta>0, \eta>0$, if the numbers $\eta=\eta(\beta, l)$ and $\delta=\delta(\beta, l)$ are appropriately chosen. We have already proved in Proposition A that $\mu(A(\beta))<1-\delta$, where $\mu$ is the (weak) limit of the measures $\mu_{n}$. Moreover, this statements also holds for the closure $\bar{A}(\beta)$ of the set $A(\beta)$ with a possibly smaller parameter $\eta$. Since $\mu_{n} \Rightarrow \mu$, $\lim \sup _{n \rightarrow \infty} \mu_{n}(\bar{A}(\beta)) \leq \mu(\bar{A}(\beta))$. This implies that also the relation $\mu_{n}(A(\beta))<1-\delta$ holds for large $n$. Proposition B is proved.

\section{The proof of the main results.}

Proof of Theorem 1. By Lemma $4, \log \left|S^{(k)}(n)\right|-\log \left|\bar{S}^{(k)}(n)\right| \Rightarrow 0$, where $\bar{S}^{(k)}(n)$ is defined in (2.12) and $\Rightarrow$ denotes stochastic convergence. Hence $S^{(k)}(n)$ can be replaced by $\bar{S}^{(k)}(n)$ in the proof of Theorem 1 .

We claim that

$$
\begin{gathered}
\frac{U_{1}(n)}{\sqrt{n}} \Rightarrow 0, \quad \frac{T_{1}^{2}(n)}{\sqrt{n}} \Rightarrow 0 \quad \text { and } \\
\frac{1}{\sqrt{n}} \log \left|\cos \left(n B_{0}(n)+T_{0}(n)-U_{2}(n)-\frac{\omega(n)}{2}\right)\right| \Rightarrow 0 .
\end{gathered}
$$

The third relation in (6.1) is needed only in the case when $0<\varphi\left(\alpha^{*}\right)<\pi$. The first two relations in (6.1) are trivial, since the random variables $U_{1}(n)$ and $T_{1}^{2}(n)$ are stochastically bounded. They are even stochastically convergent. The third relation holds, since the random variables $T_{0}(n)-U_{2}(n) \bmod 2 \pi$ converge in distribution to the uniform distribution in $[0,2 \pi)$. Indeed, by Proposition $\mathrm{B}$ the random vectors $\left(T_{0}(n), U_{2}(n)\right)$ converge in distribution to a random vector $(T, U)$, where $T$ and $U$ are independent and $T$ is uniformly distributed in $[0,2 \pi)$. Hence the random variables $T_{0}(n)-U_{2}(n) \bmod 2 \pi$ converge in distribution to the uniform distribution of $[0,2 \pi)$, as we claimed. This relation implies that the random variables $\log \mid \cos \left(n\left(B_{0}(n)-U_{2}(n)-\omega(n) / 2\right) \mid\right.$ converge in distribution to a random variable $\log |\cos V|$, where $V$ is uniformly distributed in $[0,2 \pi)$. This implies that the third relation also holds in (6.1). The random variables $S_{0}(n)$ converge to a normal law with expectation zero and variance $\operatorname{Var} \eta$, and a slight refinement of the previous argument also shows that the vectors

$$
\left(\log \cos \left(n\left(B_{0}(n)-U_{2}(n)-\frac{\omega(n)}{2}\right), S_{0}(n)\right)\right.
$$

converge in distribution to a random vector $(\log \cos V, Z)$, where $V$ and $Z$ are independent random variables, $V$ is uniformly distributed in $[0,2 \pi]$, and $Z$ is 
normally distributed with expectation zero and variance Var $\eta$. Relation (2.13) follows from the above observations. Because of Lemma 4, the form of $\bar{S}^{(k)}(n)$ defined in (2.12) and the limit behavior of the expression in the second relation of (6.1), the sign of $S^{(k)}(n)$ also satisfies the relations given in Theorem 1 .

Proof of Lemma 5. The random variable $\eta=\eta\left(\alpha^{*}\right)$ is constant if and only if

$$
\xi^{2}+2 r\left(\alpha^{*}\right) \xi \cos \bar{\varphi}\left(\alpha^{*}\right)=\text { const. } \quad \text { with probability } 1 \text {. }
$$

Since $\xi$ is a nonconstant random variable, and its values satisfy an equation of second order, its distribution is concentrated in two points $x_{1}$ and $x_{2}$ which satisfy the identity $x_{1}^{2}+2 r\left(\alpha^{*}\right) x_{1} \cos \bar{\varphi}\left(\alpha^{*}\right)=x_{2}^{2}+2 r\left(\alpha^{*}\right) x_{2} \cos \bar{\varphi}\left(\alpha^{*}\right)$, or equivalently $x_{1}+x_{2}+2 r\left(\alpha^{*}\right) \cos \bar{\varphi}\left(\alpha^{*}\right)=0$. In case (a) when the relation $0<\bar{\varphi}\left(\alpha^{*}\right)<\pi$ holds, by Lemma 1 the identity $E\left(\xi / r^{2}\left(\alpha^{*}\right)+\xi^{2}+2 r\left(\alpha^{*}\right) \xi \cos \bar{\varphi}\left(\alpha^{*}\right)\right)=0$ must hold. This is equivalent to the relation $p x_{1}+q x_{2}=0$ with $p=P\left(\xi=x_{1}\right), q=$ $P\left(\xi=x_{2}\right)=1-p$, since $r^{2}+x_{1}^{2}+2 r x_{1} \cos \bar{\varphi}=r^{2}+x_{2}^{2}+2 r x_{2} \cos \bar{\varphi}$ in this case. Finally, the second equation of the fixed point equation (1.4) $\left.r(\partial H / \partial r)\right|_{r=r\left(\alpha^{*}\right)}=$ $\alpha^{*}$ yields that $E\left(r \xi \cos \bar{\varphi}+r^{2} / r^{2}+\xi^{2}+2 r \xi \cos \bar{\varphi}\right)=\alpha^{*}$. This is equivalent to $\left(r^{2} / r^{2}-x_{1} x_{2}\right)=\alpha^{*}$, since in this case $r^{2}+\xi^{2}+2 r \xi \cos \bar{\varphi}=r^{2}-x_{1} x_{2}$, as the calculation $r^{2}+\xi^{2}+2 r \xi \cos \bar{\varphi}=r^{2}+\left(p x_{1}^{2}+q x_{2}^{2}\right)=r^{2}+\left(p x_{1}+q x_{2}\right)\left(x_{1}+x_{2}\right)-x_{1} x_{2}=$ $r^{2}-x_{1} x_{2}$ shows.

We have proved that the distribution of the random variable $\xi$ must be concentrated in two different points, and the above equations make it possible to calculate $r\left(\alpha^{*}\right)$ and $\bar{\varphi}\left(\alpha^{*}\right)$ from $\alpha^{*}$. To decide whether we get a real solution for a pair $\left(F, \alpha^{*}\right)$ we have to check whether the condition $\mid \cos \left(\varphi\left(\alpha^{*}\right) \mid<1\right.$ is satisfied. Some calculation shows that

$$
\cos \bar{\varphi}\left(\alpha^{*}\right)=-\frac{x_{1}+x_{2}}{2 r\left(\alpha^{*}\right)}=-\frac{(q-p) x_{1}}{2 q r\left(\alpha^{*}\right)}, \quad r\left(\alpha^{*}\right)^{2}=\frac{p}{q} \frac{\alpha^{*}}{1-\alpha^{*}} x_{1}^{2} .
$$

The last two identities yield that $\cos ^{2} \bar{\varphi}\left(\alpha^{*}\right)=\left((p-q)^{2} / 4 p q\right)\left(1-\alpha^{*} / \alpha^{*}\right)$. This gives that the condition $\left|\cos \bar{\varphi}\left(\alpha^{*}\right)\right|<1$ is equivalent to $\alpha^{*}>1-4 p q$.

In case (b) when the relation $\bar{\varphi}\left(\alpha^{*}\right)=0$ holds, the random variable $\xi$ is concentrated in two points $x_{1}, x_{2}, x_{1}+x_{2}+2 r\left(\alpha^{*}\right)=0$, and $E\left(\xi /(r+\xi)^{2}\right) \geq 0$. The latter relation is equivalent to $E \xi \geq 0$ in the present case. Since $2 r\left(\alpha^{*}\right)=$ $-\left(x_{1}+x_{2}\right)$ the second part of the fixed point equation (1.4) yields that $\alpha^{*}=$ $E(r / r+\xi)=-\left((p-q)\left(x_{1}+x_{2}\right) / x_{1}-x_{2}\right)$. The conditions $p x_{1}+q x_{2} \geq 0, x_{1}+$ $x_{2}<0$ are satisfied. The last condition appears, because it is equivalent to $r\left(\alpha^{*}\right)>0$. Some calculation shows that under such conditions the relation $0<\alpha^{*}<1$ also holds. Case (c) in Lemma 5 when $\bar{\varphi}\left(\alpha^{*}\right)=\pi$ can be handled similarly to case (b). Lemma 5 is proved.

Proof of TheOREM 2. Because of Lemma 4 the random variable $S^{(k)}(n)$ can be replaced by $\bar{S}^{(k)}(n)$ defined in the first line of (2.12) in the proof of the 
limit theorem. Moreover, under the conditions of Theorem $2, \sqrt{n} S_{0}(n)=0$; that is, this term is missing from (2.12). Proposition B implies that the random vectors,

$$
\left(-U_{1}(n), n B_{0}+T_{1}-U_{2}-\frac{\omega}{2} \bmod 2 \pi\right)
$$

converge in distribution to a random vector $(U, Z)$, where $Z=Z_{1}-U_{2}+$ const. $\bmod 2 \pi$ with $U_{2}=\left(-B_{2}\left(S^{2}-T^{2}\right)+2 A_{2} S T / 2\left(A_{2}+B_{2}^{2}\right)\right), U_{1}=-U=$ $-\left(A_{2}\left(S^{2}-T^{2}\right)+2 B_{2} S T / 2\left(A_{2}+B_{2}^{2}\right)\right),(S, T)$ is a Gaussian random vector with expectation zero and covariance matrix given in (2.14), the random variable $Z_{1}$ is uniformly distributed in $[0,2 \pi)$, and it is independent of the vector $(S, T)$. These relations imply that the random variable $Z$ is also uniformly distributed in $[0,2 \pi)$, and it is independent of the vector $(S, T)$ hence also of the random variable $U$, since its conditional distribution under the condition $S=x, T=y$ is the uniform distribution on $[0,2 \pi)$ for all $x$ and $y$. Lemma 4 together with the convergence of the random vectors defined in (6.2) in distribution to the random vector $(U, Z)$ imply Theorem 2 .

ProOF OF THEOREM $2^{\prime}$. Here again the investigation of the random variable $S^{(k)}(n)$ can be replaced by that of $\bar{S}^{(k)}(n)$ defined in the second line of (2.12). We are interested in the asymptotic behavior of the expression in the exponent of this formula. We describe the central limit theorem for the random vector $\left(L_{n}^{-1} S_{0}(n), T_{1}(n)\right)$ with the definition of an appropriate normalization $L_{n}$.

We have $\sqrt{n} S_{0}(n)=\sum_{j=1}^{n}\left(\eta_{j}^{(0)}-E \eta_{j}^{(0)}\right)$ with $\eta_{j}^{(0)}=\log \left|r(\alpha(n))+\xi_{j}\right|$. Under the conditions of Theorem $2^{\prime} \lim _{n \rightarrow \infty} \operatorname{Var} \eta_{j}(n)=0$, but to determine the right norming $L_{n}$ we need a sharper estimate on this variance. To get it, observe that $r(\alpha(n))=r\left(\alpha^{*}\right)+\left(\alpha(n)-\alpha^{*}\right) r^{\prime}\left(\alpha^{*}\right)+O\left(\left(\alpha(n)-\alpha^{*}\right)^{2}\right)$, and since $x_{1}+x_{2}+2 r\left(\alpha^{*}\right)=0, \eta_{j} \sim \log \left|\xi_{j}-\left(\left(x_{1}+x_{2}\right) / 2\right)+r^{\prime}\left(\alpha^{*}\right)\left(\alpha(n)-\alpha^{*}\right)\right|$. Hence $\eta_{j}$ takes two values $y_{1}$ and $y_{2}$ with probabilities $p$ and $q$, and $\left|y_{1}-y_{2}\right|=\left(4 r^{\prime}\left(\alpha^{*}\right)\left|\alpha(n)-\alpha^{*}\right| / x_{1}-x_{2}\right)(1+o(1))$, where $x_{1}>x_{2}$. We get with the help of some calculation from the second relation in (1.4) and the relations $\bar{\varphi}(\alpha)=0$ in a small neighborhood of $\alpha^{*}$ that $r^{\prime}\left(\alpha^{*}\right) E\left(\xi /(r+\xi)^{2}\right)=1$. Because of this identity and the relation $x_{1}+x_{2}+2 r\left(\alpha^{*}\right)=0$ that $r^{\prime}\left(\alpha^{*}\right)=$ $\left(\left(x_{1}-x_{2}\right)^{2} / 4\left(p x_{1}+q x_{2}\right)\right)$. Hence $\operatorname{Var} S_{0}(n)=\operatorname{Var} \eta_{j}=p q\left(y_{1}-y_{2}\right)^{2} \sim$ $p q\left(\alpha(n)-\alpha^{*}\right)^{2}\left(x_{1}-x_{2}\right)^{2} /\left(p x_{1}+q x_{2}\right)^{2}$. On the other hand, some calculation yields that $\operatorname{Var} T_{1}(n)=\left(x_{1}+x_{2}\right)^{2} /\left(x_{1}-x_{2}\right)^{2}$. Since the random variables $\xi_{j}$ take two values, the random variables $S_{0}(n)$ and $T_{1}(n)$ are linear transform of each other. Because of the above observations and the central limit theorem the random vectors $\left(L_{n}^{-1} S_{0}(n), T_{1}(n)\right.$ converge in distribution to a vector $\left(V,\left(\left(x_{1}+x_{2}\right) /\left(x_{1}-x_{2}\right)\right) V\right)$ with the choice $L_{n}=\sqrt{p q}\left|\alpha(n)-\alpha^{*}\right|\left(x_{1}-x_{2}\right) / p x_{1}+q x_{2}$, where $V$ is a standard normal random variable. This limit theorem together with the form of the second line in (2.12) imply Theorem $2^{\prime}$. 
Acknowledgement. The author thanks the referee for some useful remarks which helped to simplify certain technical details. In particular, the formulation and proof of Lemma A and some simplification in the proof of Lemmas 2 and 3 were based on the referee's ideas.

\section{REFERENCES}

[1] Dynkin, E. B. and Mandelbaum, A. (1983). Symmetric statistics, Poisson processes and multiple Wiener integrals. Ann. Statist. 11 739-745.

[2] HalÁsz, G. and SzÉKely, G. J. (1976). On the elementary symmetric polynomials of independent random variables. Acta Math. Acad. Sci. Hungar. 28 397-400.

[3] Móri, T. F. and SzÉKely, G. J. (1982). Asymptotical behavior of symmetric polynomial statistics. Ann. Probab. 10 124-131.

[4] Parthasarathy, K. R. (1967). Probability Measures on Metric Spaces. Academic Press, New York.

[5] RAUGi, A. (1979). Théorème de la limite centrale pour un produit semi-direct d'un groupe de Lie résoluble simplement connexe de type rigide par un groupe compact. Probability Measures on Groups. Lecture Notes in Math. 706 257-324. Springer, Berlin.

[6] VAN Ess, G. (1986). On the weak limit of elementary symmetric polynomials. Ann. Probab. $14667-695$.

MATHEMATiCAL INSTITUTE

OF THE HungaRIAN ACADEMY OF SCIENCES

POB 127, H - 1364

BUDAPEST

HUNGARY

E-MAIL: major@math-inst.hu 